\title{
Gut Parabacteroides merdae protects against cardiovascular damage by increasing commensal bacteria-driven branched-chain amino acid catabolism
}

\section{Shanshan Qiao}

CAS

Chang Liu

CAS

Li Sun

CAS

Tao Wang

CAS

Huanqin Dai

CAS

Wang Kai

Bao Li

CAS

Hantian Li

CAS

Shuang-jiang Liu

CAS

Hongwei Liu ( $\sim$ liuhw@im.ac.cn )

CAS

\section{Article}

Keywords: gut microbiota, Parabacteroides merdae, branched-chain amino acids, atherosclerosis, mTORC1, branched short-chain fatty acids, BCAA, BSCFA

Posted Date: December 9th, 2021

DOI: https://doi.org/10.21203/rs.3.rs-1127540/v1

License: (c) (i) This work is licensed under a Creative Commons Attribution 4.0 International License. Read Full License 
Version of Record: A version of this preprint was published at Nature Metabolism on October 17th, 2022. See the published version at https://doi.org/10.1038/s42255-022-00649-y. 


\section{Abstract}

Atherosclerosis is a chronic inflammatory disease of arteries featured with accumulated lipids, becoming the primary cause of cardiovascular diseases and death. Branched-chain amino acids (BCAAs) accumulation is defined as biomarkers of cardiometabolic diseases. Here, we revealed metabolic benefits of a previously reported gut microbiota-modulator (GMD) on atherosclerosis in $\mathrm{ApoE}^{-/-}$mice, and identify a gut symbiont Parabacteroides merdaedriven BCAA catabolism beneficial for the alleviation of atherosclerosis lesions. We also show that the porA gene responsible for the conversion of BCAAs into branched short-chain fatty acids is required for the in vivo efficacy of $P$. merdae. Furthermore, the downregulation of BCAA-activated plaque mammalian target of rapamycin complex 1 (mTORC1) pathway is suggested as the mechanism underlying the benefits of $P$. merdae. Our results demonstrate the critical role of the commensal bacteria-driven BCAA catabolism in maintaining the host cardiovascular health and supporting the gut microbiota-targeted therapeutic strategy for cardiometabolic diseases.

\section{Introduction}

Cardiovascular disease (CVD) has become increasingly prevalent and results in the death of over 17 million people each year all over the world. ${ }^{1}$ Atherosclerosis is a leading cause of CVD, and is characterized by the accumulation of lipids and fibrous plaque on vessel walls, endothelial dysfunction, and vascular inflammation in the arteries. ${ }^{2}$ The pathogenesis of atherosclerosis is complex but has been proven to involve interactions between inflammation, extracellular matrix disorders, and dyslipidemia. 3,4 Obesity, smoking, diabetes mellitus, hypertension, disturbed sleep, physical inactivity, environmental stress, and family history have all been defined as risk factors of consideration in the occurrence and development of atherosclerosis. Accordingly, current treatments against atherosclerosis in clinics mainly involve lipid- or glucose-lowering agents, healthy lifestyle, and anti-inflammatory agents. ${ }^{5}$

The recent achievements of the human genome, metabolome and microbiome have enhanced our understanding of atheroclerosis. ${ }^{6-9}$ The crucial role of the gut microbiota in CVD is being explored and elucidated. ${ }^{10}$ Some gut microbiota-derived metabolites have shown anti- or pro-atherogenic effects. ${ }^{11}$ For example, trimethylamine-N-oxide (TMAO) synthesized by gut bacteria from trimethylamine (TMA) was shown to accelerate the formation of atherosclerotic lesions. ${ }^{12}$ Short-chain fatty acids (SCFAs) that reduce insulin resistance and secondary bile acids that correct lipid and cholesterol disorders are beneficial for the prevention and amelioration of atherosclerosis. ${ }^{13,14}$

Novel anti-atherosclerosis therapies targeting gut microbiota can be expected as we gain a better understanding of the roles of gut microbiota in the progression of atherosclerosis. In our ongoing search for hypoglycemic and hypolipidemic agents, a Ganoderma-meroterpene derivative (GMD) showed gut microbiota-dependent benefits on insulin resistance, reductions in blood glucose and lipids, and prevention of hepatic steatosis in mice and rats fed a high-fat diet (HFD). ${ }^{15,16}$ Remarkably, several gut bacterial species which have therapeutic effects against obesity, hyperglycemia and hyperlipidemia, 
including Faeciroseburia intestinalis, Parabacteroides distasonis, and Bacteroides xylanisolvens, were found to be significantly enriched in the gut microbiota of animals treated with GMD. ${ }^{16-18}$ Insulin resistance, hyperlipidemia, and hepatic steatosis not only co-occur with the development of atherosclerosis, but also drive the progression of atherosclerosis. ${ }^{19-21}$ Accordingly, we hypothesized that GMD could have beneficial effects against atherosclerosis through modulating the gut microbiota.

Here, we found that GMD efficiently reduced cardiovascular risks and slowed the progression of atherosclerosis in HFD-fed $A p o E^{-/-}$mice. The highly enriched gut symbiont Parabacteroides merdae by GMD was confirmed to play a causal anti-atherogenic role. Both GMD treatment and colonization with live $P$. merdae significantly altered the functions of gut microbiota, especially enhancing the degradation of the BCAAs, leucine, isoleucine and valine. Correspondingly, the increase in branched-short chain fatty acids (BSCFAs) such as isobutyrate, 2-methylbutyrate, and isovalerate, that are exclusively derived from BCAAs by gut bacteria were detected in the feces of GMD- or $P$. merdaetreated mice. Aberrant metabolism of BCAAs and the accumulation of BCAAs in circulation were closely associated with the development of insulin resistance and CVD. ${ }^{22-24}$ By comparing mice colonized with wild-type P. merdae versus a mutant $(\triangle p o r A)$ strain deficient in BCAA degradation, we confirmed that bacteria-dependent BCAA catabolism helped to prevent atherosclerosis. The anti-atherogenic effect of $P$. merdae in the aorta was ascribed to its regulation of BCAA activated by the mTORC1 pathway. The current study confirmed the therapeutic efficacy of GMD and $P$. merdae in atherosclerosis and revealed an important role for gut symbiont-driven BCAA catabolism in maintaining the cardiovascular health of the body.

\section{Results}

\section{Alleviation of HFD-induced atherosclerosis in $\mathrm{ApoE}^{-/-}$mice by GMD}

To evaluate the effects of GMD on the progression of atherosclerosis, HFD-fed $A p o E^{-1}$ mice were grouped and orally treated with GMD (5 mg/kg or $10 \mathrm{mg} / \mathrm{kg})$, atorvastatin $(10 \mathrm{mg} / \mathrm{kg})$, or vehicle, respectively, for a period of nine weeks. The normal chow diet-fed $\mathrm{ApoE}^{+/+}(\mathrm{C} 57 / \mathrm{BL} 6$ background) mice were used as control (Fig. 1a).

Daily oral gavage with GMD prevented the progression of atherosclerosis in the HFD-fed $\mathrm{ApoE}^{-/-}$mice. By the end of nine weeks' treatment, the plasma lipid levels were significantly improved by GMD. In comparison with the vehicle-treated $\mathrm{ApOE}^{-/-}$mice, a decrease in the levels of total cholesterol (T-C, $28 \%$ and $38 \%$ ) as well as low-density lipoprotein cholesterol (LDL-C, $25 \%$ and $53 \%$ ), and a reduction of $35 \%$ and $47 \%$ in the level of total triglycerides (T-TG) was achieved by GMD at doses of 5.0 and $10.0 \mathrm{mg} / \mathrm{kg}$, respectively (Fig. 1b). A further analysis of plasma lipoprotein fractions by fast protein liquid chromatography (FPLC) indicated a pronounced increase of triglycerides in the fractions of VLDL, IDL, and $\mathrm{LDL}$, while GMD or atorvastatin treatment significantly lowered triglyceride contents in these fractions (Fig. 1c). Treatment with 5.0 and $10.0 \mathrm{mg} / \mathrm{kg} \mathrm{GMD}$ reduced plasma free fatty acid (FFA) levels by $21 \%$ and $27 \%$, respectively (Fig. 1 d). The generation of oxidized low density lipoprotein (ox-LDL) and 
its end products, malondialdehyde (MDA), high sensitivity C-reactive protein (hs-CRP), and

lipopolysaccharide (LPS) have been implicated in the initiation and progression of atherosclerosis. ${ }^{25-27}$ The levels of plasma ox-LDL and MDA, hs-CRP, and LPS were significantly reduced by $29 \%$ and $34 \%, 22 \%$ and $32 \%, 28 \%$ and $27 \%$, and $31 \%$ and $39 \%$, respectively, in the GMD-treated groups compared to control mice (Fig. 1e-h). For the inflammatory cytokines, levels of TNF- $\alpha$ and IL-1 $\beta$ were significantly decreased by GMD (Fig. 1i). In contrast, atorvastatin showed smaller effects on ox-LDL, MDA, hs-CRP, LPS, TNF-a and IL-1 $\beta$ (Fig. 1e-i). The cumulative food intake was not significantly different among the four $A p o E^{/-}$ mice groups (Extended Data Fig. 1a). Treatment with GMD also prevented weight gain and improved body composition in $\mathrm{ApOE}^{-/-}$mice (Extended Data Fig. 1b-e). The blood flow and thickness of the aortic arch branches and cardiac outflow tract were determined by color Doppler ultrasound biomicroscopy, and both GMD-treated groups showed significant improvement in these indices, compared to controls (Fig. 1j). The severity of atherosclerotic lesions was indicated by MOVAT and H\&E staining of aortic root regions and oil red $\mathrm{O}$ staining of longitudinally opened aortas. Compared to $A p O E^{+/ t}$ mice, a 3.6-fold increase in lesion area and a 2.3-fold increase in lesion size were shown in the model $\mathrm{ApoE}^{/-}$mice. Treatment with GMD substantially reduced the aortic root lesion and total aortic lesion area by $36 \%$ and $41 \%$ at the dose of $10 \mathrm{mg} / \mathrm{kg}$ (Fig. 1k, I), respectively. Collectively, GMD showed promising antiatherosclerosis efficacy by inhibiting inflammation and improving dyslipidemia.

\section{GMD modulates the composition and function of gut commensal bacteria}

In our previous reports, GMD was proven to be a powerful a-glucosidase inhibitor that retarded the decomposition of carbohydrates (like acarbose does) in the gut and changed the distribution of carbohydrates along the digestive tract, thus contributing to modifications in the gut microbiota. ${ }^{15,28}$ In this study, we analyzed the effect of GMD on the gut microbiome of HFD-fed $A p o E^{-/-}$mice by V3-V4 sequencing of $16 \mathrm{~S}$ rRNA genes. There was no significant difference between the two groups in a diversity, as indicated by observed species, Shannon and Chao1 indices (Extended Data Fig. 2a, b). The $\beta$ diversity analysis showed significant differences in the composition and abundance of the gut microbiota between GMD-treated and vehicle-treated groups ( $P=0.001$, Fig. 2a, b). Data treatment by the linear discriminant analysis (LDA) effect size (LEfSe) method revealed that the vehicle group was characterized by the families, Deferribacteraceae and Desulfovibrionaceae, the genera, Ruminiclostridium, Bilophila, Anaerotruncus, and Oscillibacter, and Helicobacter bilis at the species level. The families, Tannerellaceae and Akkermansiaceae, the genera Parabacteroides, Akkermansia, and Roseburia, and the species, Parabacteroides merdae, Bacteroides acidifaciens, and Bacteroides intestinalis were increased in the gut of GMD-treated $\mathrm{ApOE}^{-/-}$mice (Fig. 2c, d). To determine the functional changes in gut microbiota, the LEfSe was applied to the relative abundance of KEGG pathways analyzed by phylogenetic reconstruction of unobserved states 2 (PICRUSt2) (Fig. 2e, f). ${ }^{29}$ Significant enhancement of branched-chain amino acid degradation and amino acid metabolism (phenylalanine and cyanoamino acid) was predicted in the gut microbiota of GMD-treated mice. The SIMPER analysis was used to quantify the contribution of the top eight significantly altered species to alterations between the two groups. As a result, $P$. merdae enriched 420-fold by GMD was identified as the largest contributor to the induced changes in the gut microbiota by 
GMD (Fig. 2g). Subsequent analysis by co-occurrence network, Sankey diagram, and heatmap confirmed the highest enrichment of gut bacteria in the genus, Parabacteroides (Fig. 2h and Extended Data Fig. 2c, d). Next, we compared the gut microbial profiles between healthy people and patients diagnosed with CVD through a human gut metagenomes database. ${ }^{30} \mathrm{~A}$ significant decrease in the relative abundance of P. merdae was noted among the CVD patients (Extended Data Fig. 3). Based on the above data, we propose that $P$. merdae is crucial for mediating the anti-atherosclerosis effect of GMD and a key gut regulator of cardiovascular health.

\section{Gavage with live P. merdae attenuates the formation of atherosclerotic plaque in HFD-fed ApoE ${ }^{-/-}$mice}

To further clarify the role of $P$. merdae in reducing atherosclerosis, we administered live $P$. merdae (PM), heat-killed $P$. merdae (KPM) or vehicle (PBS) to HFD-fed $A p o E^{-1-}$ mice by oral gavage for four weeks (Fig. 3a). P. merdae significantly improved the signs of atherosclerosis in $A p o E^{-/-}$mice, lowering the HFDinduced weight gain by $27 \%$ (Fig. 3b), the plasma levels of TC by $22 \%$, TG by $46 \%$, and LDL-C by $19 \%$ (Fig. 3c). Bacterial treatment also reduced the aortic root lesion area by $24 \%$ and fat deposition in the plaque area by $63 \%$ (Fig. 3d), and decreased plasma LPS, ox-LDL, and circulating hs-CRP (Fig. 3e-g). Treatment with heat-killed $P$. merdae did not ameliorate the weight gain, hyperlipdemia, or lesion area, but did reduce the plasma hs-CRP to a certain extent (Fig. 3b-g), possibly because some components in the cells of $P$. merdae had anti-inflammatory effects. Gut colonization of $P$. merdae was determined post three days after the four-week treatment by qPCR. A large significant increase in the relative abundance of $P$. merdae was detected in the feces of $A p o E^{-/-}$mice treated with $P$. merdae $(0.18 \%)$ in comparison with vehicle-treated mice $(0.026 \%)$ (Fig. $3 \mathrm{~h})$. With regard to the overall composition of the gut microbiota, there was no significant difference between $P$. merdae treatment and vehicle in a diversity (Extended Data Fig. 4a). The $\beta$ diversity analysis showed a significant difference in the OTUs at the genus and species level in the gut microbiota between $P$. merdaetreated and vehicle-treated $A p o E^{1-}$ mice $(P=0.001$, Fig. 3i, Extended Data Fig. 4b-c). Consistent with the results of GMD treatment (Fig. 2d, e), P. merdae colonization significantly increased the abundance of Akkermansia and Parabacteroides in $\mathrm{ApoE}^{-/-}$mice (Fig. 3j, k, Extended Data Fig. 4d-e), and functionally enhanced the degradation of valine, leucine and isoleucine as predicted by KEGG pathway analysis (Fig. 3l). Based on the above results, we conclude that modulation of gut microbiota by $P$. merdae administration contributes to its beneficial effects on atherosclerosis.

Next, we investigated whether live $P$. merdae exhibited anti-atherosclerotic effects in $A p o E^{-/-}$mice treated with broad-spectrum antibiotics (Abx) to reduce the gut bacterial load (Extended Data Fig. 5a). Abxtreated $A p o E^{-1-}$ mice were gavaged with $2 \times 10^{8} \mathrm{CFU}$ of live $P$. merdae or vehicle daily. In comparison with pseudo germ-free control $A p o E^{-/-}$mice, $P$. merdae treatment resulted in improvements in body weight, hyperlipidemia, hyperglycemia, and atherosclerosis biomarkers (ox-LDL, MCP-1, TNF-a, IL-1 $\beta$ ) (Extended Data Fig. 5b-p).

These findings confirmed the capability of the gut symbiont $P$. merdae to protect $A p o E^{/-}$mice against atherosclerosis, supporting the potential of human gut-derived $P$. merdae as a probiotic with therapeutic 
potency.

\section{Involvement of branched-chain amino acid degradation pathways in the anti-atherosclerotic effects of $\mathrm{P}$.} merdae

Plasma BCAAs have been identified as high-risk factors for the occurrence and development of CVD. ${ }^{31-34}$ Transplantation of gut microbes from obese people into germ-free mice demonstrated the role of gut microbiota in adjusting the level of circulating BCAAs in the host. ${ }^{35}$ It is known that BCAAs can be transformed into different BSCFAs in the gut. ${ }^{36}$ Since BCAA degradation in the gut was predicted to be enhanced in both GMD-treated and live P. merdaeinoculated $A p o E^{-/-}$mice (Fig. 2e and $3 \mathrm{l}$ ), we measured BCAAs and BSCFAs levels in fecal samples. A significant increase in BSCFAs including isobutyrate, isovalerate, and 2-methylbutyrate along with a corresponding decrease in BCAAs was observed (Fig. 4a, b), which confirmed that $P$. merdae could regulate BCAA degradation in the gut.

To confirm P. merdae's capability of degrading BCAAs, we analyzed the levels of BSCFAs in cultures. Isobutyrate, isovalerate, and 2-methylbutyrate were detected after $24 \mathrm{~h}$ incubation, further supporting the ability of $P$. merdae to convert BCAAs into BSCFAs (Fig. 4c and Extended Data Fig. 6). To determine the BCAA-related metabolic pathways in $P$. merdae, we performed pangenomic profiling using gutSMASH to identify a wide range of known and putative gene clusters. ${ }^{37}$ The porA gene was reported to catalyze the transformation of BCAAs into BSCFAs in Clostridium sporogenes, and using it as a query sequence, we identified a putative BCAA degradation sequence in the $P$. merdae genome with $60 \%$ similarity to porA (Extended Data Fig. 7). We also compared the porA gene repertoire in a cohort of 218 Chinese individuals with atherosclerotic CVD vs 187 healthy controls (Supplementary Table 1). ${ }^{38}$ We found that atherosclerosis patients harbored lower abundance of both the porA gene $(P<0.0001$; Extended Data Fig. 8a) and $P$. merdae $(P<0.05$; Extended Data Fig. 8b) than healthy people, supporting our hypothesis that the porA gene and the commensal $P$. merdae were instrumental in maintaining cardiovascular health. Further analysis of the porA gene abundance in different taxonomic groups across the 405 metagenomes showed that the number of porA reads per metagenome $\left(R^{2}=0.261, P<0.0001\right)$ were positively associated with the relative abundance of $P$. merdae (Extended Data Fig. 8c). The porA gene harboring in the $P$. merdae is supposed to have important physiological functions.

To test this supposition, we knocked out the porA gene in P. merdae (Extended Data Fig. 9a). GC-MS analysis indicated that the mutant strain had lost the capacity to convert BCAAs into BSCFAs (Fig. 4d). Next, to test whether the porA gene-dependent BCAA degradation was essential for $P$. merdae's beneficial anti-atherosclerotic properties, we inoculated HFD-fed $\mathrm{ApoE}^{-/}$mice with the $P$. merdae mutant strain lacking the porA gene (PM $\triangle \mathrm{PorA})$ or the wild type strain (PMWT) and compared their effects on atherosclerosis lesions (Fig. 5a). The deficiency of the porA gene in P. merdae did not affect its ability to colonize the mouse gut, as indicated by qPCR analysis (Fig. 5b). As expected, the plasma and fecal BCAAs levels were higher in PM $\triangle$ PorA -treated mice than in the PMWT-inoculated group (Fig. 5c, d). In comparison with the notable reduction in atherosclerosis with the WT P. merdae strain, HFD-fed ApoE ${ }^{-/}$ 
mice orally treated with PM $\triangle$ PorA exhibited no improvements in aortic root lesion area, fat deposition in plaques, body weight, plasma lipids (T-TC, T-TG, LDL-C), LPS, ox-LDL, and hs-CRP (Fig. 5e-k). To exclude the host impact on BCAA degradation, we compared the expression difference of key BCAA catabolic genes including BCAT2, BCKD subunits E1a, E1 $\beta$ and E2, as well as the BCKD phosphatase PP2Cm in liver, fat, and muscle between the two groups and there was no difference in the expression of these genes. (Extended Data Fig. 10a-c). Thus, we may conclude that the gut symbiont, $P$. merdae, exerts its protective effects on atherosclerosis through degradation of intestinal BCAAs.

\section{Benefits of P. merdae are related to inhibition of the BCAA-activated plaque mTORC1 signaling pathway}

Insulin resistance is now recognized as being strongly associated with the occurrence and development of atherosclerosis. A line of evidence showed a positive correlation between elevated plasma BCAAs and insulin resistance. ${ }^{31}$ In the present work, significant reduction of hyperglycemia and improvement in insulin sensitivity was observed in HFD-fed $\mathrm{ApoE}^{-/}$mice that were orally inoculated with wild type $P$. merdae possessing strong BCAA degradation ability (Fig. 6a-h). In contrast, the $P$. merdae mutant strain deficient in BCAA degradation did not lower plasma glucose levels or improve insulin sensitivity (Fig. 6ah). Recent studies have revealed that dietary restriction of BCAAs improved insulin sensitivity in insulinresistant rodent models. ${ }^{39,40}$ Insulin-resistant can drive the progression of atherosclerosis, the increased BCAA degradation by $P$. merdae appears to alleviate hyperglycemia and insulin resistance, and consequently protects against atherosclerosis.

With respect to the potential mechanism of BCAA action, over-activation of the mTORC1 pathway was associated with atherosclerosis and insulin resistance. ${ }^{41,42}$ Leucine, isoleucine and valine have been demonstrated to be mTORC1 agonists. ${ }^{43}$ In early studies, dietary protein drives atherosclerosis and lesion, especially increased circulating leucine levels and acutely activates plaque macrophage mTORC1 signalling. ${ }^{44}$ Supplementation with BCAAs was reported to activate the mTORC1 pathway exacerbating inflammation and oxidative stress in endothelia cells and inducing insulin resistance in humans. ${ }^{23,31}$ Here, we compared the mTORC1 pathway activation in atherosclerotic plaque macrophages by immunofluorescence staining of aortic roots from mice gavaged with PM $\triangle$ PorA or PMWT. Activation of mTORC1 was determined as phosphorylation of the downstream target ribosomal protein S6 (S6). ${ }^{45}$ Mice colonized with WT P. merdae showed lower plaque phospho-S6 (pS6) intensity than mice treated with the PorA-deficient mutant strain, representing less activation of the mTORC1 pathway (Fig. 6i). Based on this evidence, we concluded that regulation of the BCAA-activated mTORC1 pathway contributed to the mechanism underlying the anti-atherosclerotic efficacy of $P$. merdae.

In this study, we demonstrated that the enrichment of $P$. merdae by GMD helped to reduce the formation of atherosclerotic lesions in a mouse model by enhancing BCAA catabolism in the gut and modulating the gut microbiome. The human gut-derived $P$. merdae strain effectively degraded BCAAs and is a potential probiotic for protection against CVD. 


\section{Discussion}

Growing evidence suggests that gut microbes play a key role in the development of atherosclerosis. Drugging the microbiome, such as with DMB (3,3-dimethyl-butan-1-ol) ${ }^{46}$ or cyclic D, L-a-peptides ${ }^{47}$ has been tested and shown to be an effective strategy for treating atherosclerosis.

Here, we demonstrated the therapeutic effects of GMD and the gut commensal $P$. merdae enriched by this compound in suppressing the progression of atherosclerosis. GMD is a synthetic meroterpene derivative on the basis of ganomycin I that was isolated from several species of Ganoderma mushrooms. ${ }^{15,48}$ The therapeutic efficacies of GMD on insulin resistance, hyperglycemia, hyperlipidemia and hepatic steatosis have been validated in our earlier investigations. ${ }^{15,16}$ The multiple metabolic benefits conferred by GMD makes it a promising agent for treating metabolic diseases with complicated pathogenesis. Early studies of GMD found that it was a potent a-glucosidase inhibitor that significantly suppressed the hydrolysis of dietary polysaccharides in the upper part of the small intestine. An accumulation of polysaccharides was found in the rectum and colon of animals after treatment with GMD, which could drive changes to their gut microbiota. ${ }^{15}$ Butyrate-producing gut bacteria in the Lachnospiraceae family were increased in mice treated with GMD. ${ }^{17}$ In rats with non-alcohol-related fatty liver disease (NAFLD), GMD significantly enhanced the abundance of Bacteroides in addition to butyrate-producing gut bacteria. ${ }^{16}$ In this study, a dramatic shift in gut microbiota with a significant increase in species of the genus Parabacteroides and Bacteroides was observed in GMD-treated $A p O E^{/-}$mice. As a result of SIMPER analysis, $P$. merdae was identified as the predominant species in the gut after GMD dosing. Gut bacteria in the genus Parabacteroides, including $P$. distasonis and $P$. goldsteinii, have been shown to reduce hyperlipidemia and insulin resistance in animal tests. ${ }^{18,49} P$. merdae also had the capacity to transform 3-oxo- $\Delta^{4}$-LCA and 3oxo-alloLCA into isoalloLCA, and 3-oxo-LCA into isoLCA, which potentially contributed to protection against pathogen infections in a centenarian. ${ }^{50}$ Oral administration of a combination of Akkermansia muciniphila, $P$. merdae, and $P$. distasonis protected mice against acute electrically induced seizure. ${ }^{51}$ To date, effects of $P$. merdae on atherosclerosis lesions have not been reported. In this work, we demonstrated that a $P$. merdae strain isolated from a healthy human attenuated atherosclerotic lesions in normal $A p o E^{/-}$mice and antibiotics-pretreated $A p o E^{--}$mice. Gavage with live $P$. merdae also influenced the structure and composition of the mouse gut microbiota, especially in terms of a significant increase of Akkermansia muciniphila that was reported to protect $A p o E^{1-}$ mice against atherosclerosis. ${ }^{52}$ Cooperative interaction exists between $A$. muciniphila and $P$. merdae in an vitro crossing feeding system, could contribute to the endogenous enrichment of both in mice. ${ }^{51}$ These results suggested a complex, beneficial interaction between $P$. merdae and other symbionts in the gut, which needs expanded research to determine the mechanism.

The essential BCAAs, leucine, isoleucine, and valine, are commonly used as nutrients for fitness in athletes and to reduce the severity of liver disease. Recently, a large body of epidemiological and experimental data revealed that BCAAs were cardiometabolic risk factors. ${ }^{22-24,31-34}$ In a study of 2,000 CVD patients, the level of BCAAs and their metabolites in plasma was strongly independent associated 
with the severity of CVD. ${ }^{34}$ It was also reported that chronic accumulation of BCAAs could inhibit glucose metabolism and induce cardiac ischemic injury. ${ }^{22}$ BCAA catabolic defect was reported as a significant contributor to the pathogenesis of heart failure. ${ }^{23}$ Moreover, BCAA catabolism is an important regulator of platelet activation and is associated with arterial thrombosis risk. ${ }^{24}$ The serum levels of BCAAs are normally tightly controlled by catabolism through common biochemical mechanisms involving transamination and decarboxylation reactions. The three BCAAs are rapidly metabolized and shuttled into the tricarboxylic acid cycle in the muscle, brown fat, liver, kidneys, and heart. ${ }^{53}$ Besides hostdependent BCAA catabolism, accumulating evidence has revealed that the gut microbiota play an important part in BCAA metabolism. In one study, gut microbes from twins differing in BMI (one was obese) were characterized and the obesity-associated bacteria showed higher rates of BCAA synthesis and lower rates of BCAA catabolism. Fecal microbiota transplanted from the obese individual into germfree mice effectively enhanced their circulating BCAAs. ${ }^{35}$ In another work, an increase in plasma BCAAs in insulin-resistant individuals was found to be correlated with a gut microbe population having an enriched capacity for BCAA synthesis. The associated commensal bacterium, Prevotella copri, was shown to increase total serum BCAAs and induce insulin resistance in mice fed a high-fat diet. ${ }^{54}$ Moreover, BCAAs were found to be involved in the a-galactosylceramides biosynthesis of gut symbiont Bacteroides fragilis in vitro and vivo. ${ }^{55}$ Considering these evidences, intervention to increase BCAA catabolism is a promising therapeutic strategy against CVD.

In one more recent report, porA gene-dependent production of BSCFAs by the gut commensal Clostridium sporogenes from BCAAs was demonstrated. The porA gene and its homologs are found to be widely present in human gut metagenomes and highly transcribed in human stool metatranscriptomes. ${ }^{36}$ In the current work, $A p o E^{-/-}$mice gavaged with live $P$. merdae showed significant reductions in plasma leucine, isoleucine, and valine, compared to vehicle controls. We annotated a porA homolog in the genome of $P$. merdae and constructed a porA mutant strain. Mutation of porA functionally damaged the transformation of BCAAs into BSCFAs. Oral administration of the porA mutant strain failed to attenuate the symptoms of atherosclerosis in $\mathrm{ApOE}^{-1}$ mice compared to mice treated with the wild strain. Our current work on the gut commensal $P$. merdae provides evidence supporting a causal relationship between BCAA catabolism mediated by the gut bacterium and protection against atherogenesis in the $\mathrm{ApoE}^{/-}$mouse. Restriction of dietary isoleucine or valine intake has shown metabolic benefits on obesity, insulin resistance and CVD. ${ }^{56}$ However, as BCAAs are necessary for protein synthesis and energy production, a diet-based intervention has serious limitations in practice. Supplementation with probiotics possessing BCAA degradation ability is an alternative approach for lowering the risk of atherosclerotic lesions.

Compared to SCFAs, the physiological roles of BSCFAs are less well known. In one study, isobutyric acid or isovaleric acid were proven to suppress cAMP-mediated lipolysis and insulin-stimulated de novo lipogenesis, and promote insulin-stimulated glucose uptake in adipocytes. ${ }^{57}$ Recently, isovalerate was identified as a ligand for Olfr558, a G-protein-coupled receptor (GPCR) controlling the release of serotonin 
from enterochromaffin cells. ${ }^{58} \mathrm{An}$ increase of gut BSCFAs in germ-free mice was shown to reduce levels of surface-bound IgA in immune cell populations. ${ }^{36}$ In the current work, the level of secreted IgAs from the plaque macrophages of wild type strain-treated mice was much lower than that of mutant strain-treated mice (Extended Data Fig. 11); this could account for the BSCFA-mediated immune suppression involved in the reduction in plaque lesions. The physiological function of BSCFAs and the underlying mechanism of plaque inhibition need to be investigated in greater depth.

In summary, we provide evidence for the metabolic benefit of GMD in preventing atherosclerosis, and the discovery of a significantly enriched gut symbiont, $P$. merdae was demonstrated to play a causative role in the anti- atherosclerosis effects of GMD. The $P$. merdae enhanced BCAA catabolism was confirmed to be responsible for the observed cardiometabolic benefits. Alleviation of atherosclerosis lesions by GMD and $P$. merdae supports the potential for targeting the gut microbiome as an anti-CVD treatment strategy.

\section{Methods}

Mice. All animal procedures in this study were performed in accordance with the recommendations in the Guide for the Care and Use of Laboratory Animals of the Institute of Microbiology, Chinese Academy of Sciences (IMCAS) Ethics Committee.

Mice with less differences in body weight were randomly assigned to experimental groups, and no mice were excluded from the analysis. The 146 female mice used for this study are described in Supplementary Table 2.

8-week-old female $\mathrm{ApoE}^{-/-}$mice were fed with a high-fat diet (60 kcal\% fat, $20 \mathrm{kcal} \%$ protein and 20 kcal\% carbohydrate, Cat. D12492i, Research Diet, New Brunswick, NJ, USA) for 8 weeks, while 8-week-old female C57BL/6J as the background were fed on a normal chow diet (Lab Diet, Cat. 5001). All mice were housed in a specific pathogen-free (SPF) facility, with a strict $12 \mathrm{~h}$ light/dark cycle (8:00 a.m. to 8:00 p.m.), humidity at $50 \pm 15 \%$, a temperature of $22 \pm 1{ }^{\circ} \mathrm{C}$, and ad libitum access to food and water. The health status of mice was determined via daily observation by technicians supported by veterinary care. All animal procedures in this study were performed in accordance with the recommendations in the Guide for the Care and Use of Laboratory Animals of the Institute of Microbiology, Chinese Academy of Sciences (IMCAS) Ethics Committee.

Mice with less differences in body weight were randomly assigned to experimental groups, and no mice were excluded from the analysis. The body weight of the mice was measured by weighing the mice on a scale once three days, and the body fat content and fat ratio were measured and calculated with an MRI body fat measurement instrument (EchoMRI-700, EchoMRI) two days before sacrifice.

Animals in each group were subjected to the OGTT and an ITT test before cervical dislocation sacrifice. The blood was sampled from the portal and cava veins and plasma was collected to measure BCAAs levels and other biochemical indicators. The liver, fat, muscle, feces, cecum, and intestines were quickly 
frozen and stored at $-80{ }^{\circ} \mathrm{C}$, the whole aorta was harvested after heart perfusion and preserved in $4 \%$ paraformaldehyde. All efforts were made to minimize suffering.

In assays with GMD, HFD-fed $A p o E^{-/-}$mice were sorted into four groups, GMD-treated groups were given with GMD (10 mg/kg and $5 \mathrm{mg} / \mathrm{kg}$ ) daily by gavage. Atorvastatin-treated groups were administered 10.0 $\mathrm{mg} / \mathrm{kg}$ daily by gavage. Treatments were continued for 8 weeks.

In assays involving live $P$. merdae and heat-killed $P$. merdae (KPM), HFD-fed $A p o E^{-/-}$mice were sorted into three groups. Mice in the PM group were treated daily with $2 \times 10^{8}$ cfu of live P. merdae in $0.2 \mathrm{~mL}$ of sterile anaerobic PBS by mouth. Mice in the KPM group were given $2 \times 10^{8} \mathrm{cfu}$ of heat-killed (Pasteurization consisted of heat treatment at $70^{\circ} \mathrm{C}$ for 30 min of fresh $P$. merdae) $P$. merdae suspended in $0.2 \mathrm{~mL}$ of sterile anaerobic PBS daily. The vehicle group was given an equivalent volume of sterile anaerobic PBS. Treatments were continued for 4 weeks. In assays with live $P$. merdae and $P$. merdae $\triangle \mathrm{PorA}, \mathrm{HFD}$-fed $\mathrm{ApoE}^{-/-}$mice were sorted into three groups and using the same method as described for the PM and KPM experiments.

Microbial strains. The $P$. merdae strain was isolated from human feces by the microfluidic streak plate (MSP) method. ${ }^{59}$ The strain was identified by comparing 16S rRNA gene sequence with those in the NCBI reference database (https://www.ncbi.nlm.nih.gov/). The P. merdae strain was cultured in yeast extract, casitone and fatty acid (YCFA) medium at $37^{\circ} \mathrm{C}$ in an anaerobic chamber for 24 hours. For the in vivo efficacy assay, cell pellets of the wild-type and mutant strains of $P$. merdae were obtained by centrifuging at $8,000 \times \mathrm{g}$ for $10 \mathrm{~min}$ at $4^{\circ} \mathrm{C}$. The cell suspension for oral administration was prepared by suspending the cultured bacterial cells in oxygen-free PBS at a final cell density of $1 \times 10^{9} \mathrm{CFU} / \mathrm{mL}$ and $200 \mu \mathrm{L}$ of the bacterial suspension was given daily by gavage. The heat-killed $P$. merdae control was prepared by heating a culture at $70^{\circ} \mathrm{C}$ for $30 \mathrm{~min}$.

Imaging of arterial lesions with high frequency ultrasound. A high frequency ultrasound system (Vevo 2100, Visualsonics, Toronto, Canada) equipped with a linear array transducer (MS 550D, 22-55 MHz) was used to detect atherosclerotic lesions at the aortic sinus. Briefly, $\mathrm{ApOE}^{-1}$ mice anesthetized with isoflurane were placed on a heated procedural board and limbs were taped to electrocardiogram electrodes coated with electrode cream. A rectal thermometer was inserted to assist with maintaining normothermia $\left(37^{\circ} \mathrm{C}\right)$. The fur at the imaging location was shaved and warm ultrasound gel was liberally applied to ensure optimal image quality. The aortic sinus was imaged and visualized using a long-axis view; a CINE loop of 100 frames was stored for later off-line analysis. The time gain compensation curve was adjusted to produce uniform echo intensity. The gain was set to $30 \mathrm{~dB}$ and the dynamic range to 65 $\mathrm{dB}$. To reduce variability, image parameters were held constant throughout the experiment. All examinations were performed by an experienced operator, and measurements were repeated three times at each site.

Assessment of aortic atherosclerotic lesion areas. Aortic specimens were resected and fixed with $10 \%$ formaldehyde in phosphate-buffered saline ( $\mathrm{pH} 7.4$ ), embedded in paraffin, sectioned and stained with 
hematoxylin and eosin (H\&E), oil red O, or Masson's stain. For staining of atherosclerotic lesions in the entire aorta, the aorta-to-iliac arteries were dissected and opened along the ventral axis. The aortas were pinned onto black wax in a pan and stained with oil red O. Images of the aorta were captured with a digital camera equipped with a Canon EOS 650D lens and further analyzed using ImageJ software. For histological analysis of atherosclerotic lesions in the aortic root, sections including the aortic valve were stained with H\&E, oil red O, or Masson's stain. Quantitative analysis of lesion areas and lipid-stained lesions was performed using ImageJ software.

Fecal collection. Animals were kept in an empty cage without bedding for $15 \mathrm{~min}$ to gather fresh stool samples into tubes, which were stored at $-80^{\circ} \mathrm{C}$ until analysis.

Cecal DNA extraction, library preparation, and sequencing. Cecal DNA was extracted using the QIAamp DNA stool mini kit (Qiagen). The V3-V4 region of the bacterial 16S rRNA was amplified by polymerase chain reaction using $20 \mathrm{ng}$ of DNA, Platinum Hot Start PCR master mix (Thermo Fisher Scientific), the primers, F: ACTCCTACGGGAGGCAGCA, and R: GGACTACHVGGGTWTCTAAT, and sequenced on the HiSeq PE250 platform (Illumina) using the $2 \times 250$ bp paired-end protocol.

16S rRNA gene bioinformatics analyses. The overall amplicon sequencing data were from two experiments: (1) $\mathrm{ApoE}^{-/-}$mice treated with GMD versus vehicle, and (2) $A p o E^{-/-}$mice treated with live $P$. merdae versus vehicle (Supplementary Table 3). Using the same QIIME2 workflow, 16S data were analyzed (see Data Supplement for details). The obtained pair-end reads were trimmed and then assembled with PANDAseq. ${ }^{60}$ After filtering the chimeras by USEARCH, sequences were clustered into operational taxonomic units (OTUs) at a similarity cutoff value of $\geq 97 \%$ using the UPARSE algorithm. A representative sequence of each OTU was assigned to the taxa at genus level in the optimized version of the RDP database (http://rdp.cme.msu.edu). Each unique OTU was subjected to BLAST against the NCBI 16S rRNA database to identify the closest match to the taxa at species level based on lowest e-value and identity $97 \%$. Abundances were recovered by mapping the de-multiplexed reads to the UPARSE OTUs. A rarefied OTU table from the output files was further analyzed with a visualization toolkit. The resulting abundance table and taxonomic classification was loaded into R. Statistical analysis of differentially abundant sequences and taxa was performed using DESeq2 $1.16 .1^{61}$ and the log2 fold changes (log2FC) were obtained from comparison with the reference level.

Computational identification of genes associated with BCAA degradation. To identify and quantify the abundance of porA genes across the publicly available human gut metagenomes, the quality-control of processed reads was assessed using FASTQC (FastQC, bioinformatics.babraham.ac.uk/projects/fastqc/). Specifically, we used HUMAnN 3.0 to create an abundance profile of microbial metabolic pathways based on UniRef 90 sequences and annotations. ${ }^{62}$ Next we used Diamond BLASTp to identify the nine homologs of the porA amino acid sequence in the 405 human gut metagenomes. ${ }^{63}$ The nine query sequences consisted of experimentally characterized porA sequences identified through homology searches (Supplementary Table 1). A reference gene was considered a homolog if it was aligned with one of the six query genes with $\geq 50 \%$ amino acid identity 
(AAID) over $\geq 70 \%$ of each gene's length. Next, we obtained the relative abundance of the porA homologs across the 405 gut microbiome samples from Chinese individuals. ${ }^{38}$

Relative abundance of $\mathrm{P}$. merdae in human gut metagenomes. After quality control, the 405 human gut metagenomes were annotated by species with kraken2, ${ }^{64}$ using the kraken2_hGMB database for comparison.${ }^{59} \mathrm{Next}$, we calculated the relative abundance of $P$. merdae across the gut microbiome samples.

Biochemical and immunological assays. Levels of plasma glucose, TC, TG, HDL-C, LDL-C, HbA1C, TNF- $a$, IL-1 $\beta$, LPS, ox-LDL, hs-CRP, MCP-1, slgA and insulin were measured by commercial kits. The insulin sensitivity index (ISI) was calculated from the values of fasting blood glucose (FBG, in $\mathrm{mg} / \mathrm{dL}$ ) and fasting blood insulin ( $\mathrm{FBI}$, in $\mathrm{mU} / \mathrm{L})$. ISI = 1/1000 (FBG $\times \mathrm{FBI}$ ). An ITT was performed by injecting insulin $(0.6 \mathrm{U} / \mathrm{kg})$ intraperitoneally after $6 \mathrm{~h}$ of fasting. An OGTT was performed by giving a glucose bolus (2 $\mathrm{g} / \mathrm{kg}$ ) by gavage after overnight fasting. The level of blood glucose was measured using a glucose meter (Accu-Chek, Roche, Switzerland) before oral glucose load ( $0 \mathrm{~min}$ ) and at 40, 100, and $160 \mathrm{~min}$ after oral glucose load. The AUCs generated from the data collected during the ITT and OGTT were calculated with GraphPad 8.0.

For lipoprotein profiling, $100 \mu \mathrm{L}$ of plasma was separated by fast-performance liquid chromatography (FPLC) on a Superose S-6 10/300 GL column (GE

Healthcare, Sweden) at a flow rate of $0.5 \mathrm{~mL} / \mathrm{min}$. Forty sequential fractions of $500 \mu \mathrm{L}$ each were collected, and triglyceride concentration was measured in each fraction.

Immunofluorescence analysis. Tissue sections were blocked with $5 \%$ bovine serum albumin (BSA) for 1 $h$, and incubated with the antibodies sequentially. Primary antibodies (Goat anti-Mouse IgA Antibody HRP Conjugated, Bethyl, RRID: AB_67140; Rabbit Anti-Mouse Phospho-S6 Ribosomal Protein, Cell Signaling, RRID:AB_331679; Rat Anti Mouse CD68, AbD Serotec, RRID: AB_2074849) were used at 1:250 dilution. Species-specific fluorescent secondary antibodies [Donkey Anti-Rabbit IgG H\&L (Alexa Fluor® 647), Abcam, RRID: AB_2752244; Donkey Anti-Rat IgG H\&L (Alexa Fluor® 488), Abcam, RRID: AB_2737355; Donkey Anti-Goat IgG H\&L (Alexa Fluor® 594), Abcam, RRID: AB_2810222] were used at 1:250 dilution. An LSM 700 confocal microscope (Zeiss) was used for image acquisition; images were quantified with the Zen microscope software 2011, blue edition (Zeiss).

Real-time qPCR Analysis. Total RNA was extracted and purified from liver, fat, and muscle tissue following the protocol described in the blood and tissue kit with TRIzol reagent and RNAeasy Mini Kit (Qiagen). Quantification and integrity analysis of total RNA were performed by running $1 \mu \mathrm{L}$ of each sample on an Agilent 2100 Bioanalyzer (Agilent RNA 6000 Nano Kit, Agilent). The cDNA was prepared by reverse transcription. Real-time qPCR was conducted with Gapdh mRNA as a housekeeping gene. Sequences of the primers used for real-time qPCR are shown in the supporting information (Supplementary Table 4). The qPCR mixture contained $100 \mathrm{ng}$ of cDNA, $0.5 \mu \mathrm{M}$ primers $/ 0.15 \mu \mathrm{M}$ probe, and Kapa Fast qPCR Mix (Kapa Biosystems). PCR amplification was performed using the following 
cycling parameters: $3 \mathrm{~min}$ at $95^{\circ} \mathrm{C}, 37 \mathrm{cycles}$ of $3 \mathrm{~s}$ at $95^{\circ} \mathrm{C}$, and $30 \mathrm{~s}$ at $60^{\circ} \mathrm{C}$. The copy number was determined from the standard curve generated using a synthetic template.

Generation of a porA genedeficient P. merdae strain. An internal fragment ( $615 \mathrm{bp})$ of the porA gene was cloned into the pGERM suicide vector incorporating $E$. coli (bla) and $P$. merdae (ermG) selective markers. The resulting construct was transformed into the conjugative $E$. coli WM3064 strain, which is auxotrophic for diaminopimelic acid (DAP). The E. coli donor strain WM3064 was grown aerobically at $37^{\circ} \mathrm{C}$ in Luria broth (LB) supplemented with DAP $(100 \mu \mathrm{g} / \mathrm{ml})$ and ampicillin $(50 \mu \mathrm{g} / \mathrm{ml})$ until it reached an $\mathrm{OD}_{600}$ of 0.2. The $P$. merdae recipient was grown anaerobically at $37^{\circ} \mathrm{C}$ in YCFA medium until it reached an $\mathrm{OD}_{600}$ of 1.0. A $2 \mathrm{ml}$ mixture of equal volumes of donor and recipient cultures (1:1 ratio) was centrifuged, the supernatant was discarded and the mating mix was placed on a YCFA-medium agar plate. After aerobic overnight incubation, the plate was transferred into an anaerobic station and the bacteria were suspended in $5 \mathrm{ml}$ of gifu anaerobic medium. After $5 \mathrm{~h}$ anaerobic incubation at $37^{\circ} \mathrm{C}, 100 \mu \mathrm{L}$ of cell suspension was spread on YCFA medium agar plates supplemented (mutant selection) or not (control) with $25 \mu \mathrm{g} / \mathrm{mL}$ erythromycin. After four days of anaerobic incubation, erythromycin-resistant colonies were picked and used for genomic DNA extraction. Plasmid insertion into the target gene was then verified by PCR using primers targeting junction regions between PGERM and PorA gene (Supplementary Table 4).

High-performance gas chromatography. The extractions of BCAAs and SCFAs were performed at $4^{\circ} \mathrm{C}$. One $\mathrm{mL}$ of $5 \mathrm{mM} \mathrm{NaOH}$ containing an internal standard $\left(5 \mu \mathrm{g} / \mathrm{mL}\left[2 \mathrm{H}^{3}\right]\right.$-L-leucine, Sigma-Aldrich) was added to fecal samples (50-100 mg), and the samples were homogenized for 10 min and centrifuged at $12,000 \mathrm{r}$ at $4^{\circ} \mathrm{C}$ for $20 \mathrm{~min}$. Aliquots of $600 \mu \mathrm{L}$ of fecal homogenate were transferred into $10 \mathrm{~mL}$ glass centrifuge tubes, and $200 \mu \mathrm{L}$ of sterile DIW was added. For plasma samples, $100 \mu \mathrm{L}$ of each sample and $500 \mu \mathrm{L}$ of $5 \mathrm{mM} \mathrm{NaOH}$ containing internal standard were mixed in a $10 \mathrm{~mL}$ glass centrifuge tube. An aliquot of $500 \mu \mathrm{L}$ propanol/pyridine mixture solvent ( $\mathrm{v} / \mathrm{v}=3: 2)$ and $100 \mu \mathrm{L}$ of propyl chloroformate were subsequently added to the glass tube and vortexed briefly. The derivatization reaction was continued with ultrasonication for $1 \mathrm{~min}$. The derivatives were extracted twice with hexane and anhydrous sodium sulfate ( 10 mg) was added to remove traces of water. GC-MS analysis was performed using an Agilent $7890 \mathrm{~A}$ gas chromatography system.

DNA isolation and qPCR. Bacterial DNA was extracted from feces of mice with the QIAamp DNA stool mini kit (Qiagen), qPCR was performed on a 7500 Fast Real-Time PCR System using the primers (Supplementary Table 4).

Antibiotic treatment. Ampicillin $(1 \mathrm{mg} / \mathrm{mL})$, vancomycin $(5 \mathrm{mg} / \mathrm{mL})$, neomycin $(10 \mathrm{mg} / \mathrm{mL})$ and metronidazole $(10 \mathrm{mg} / \mathrm{mL})$ were provided in drinking water for two weeks to specific-pathogen-free (SPF) $\mathrm{ApoE}^{-/}$mice. Antibiotic-treated mice were maintained in sterile cages, given sterile food and water, and handled aseptically. 
Quantification and statistical analysis. GraphPad Prism version 8.0 (GraphPad Software, San Diego, CA) was used for statistical analysis. The experimental data are shown as the mean \pm s.e.m. The sample size was estimated on the basis of previous experience, sample availability and previously reported studies. The numbers per group in the figure legends refer to the number of mice per group. We collected data from animal studies in a blinded manner, and no data were excluded from the analysis. The normal distribution of the data was determined by the D'Agostino and Pearson omnibus normality test. For statistical comparisons, Student's t-test or one-way ANOVA with Tukey's test was used to compare normally distributed variables. Non-normally distributed data were compared by the Mann-Whitney U test (between two groups) or the Kruskal-Wallis test (among multiple groups). Spearman's correlations between changes in microbial species and host BCAAs and BSCFAs levels were calculated based on species with significant differences between the two groups. The Benjamini-Hochberg procedure with a cutoff of 0.1 was applied to all Spearman's correlations. $P<0.05$ was considered significant.

\section{Declarations}

Data availability. Raw sequence data from all 16S rRNA sequencing experiments are deposited in national microbiology data center (NMDC) at https://nmdc.cn/, sequence read archive under BioProject accession number SUB1635388067731 and SUB1635384534089. Additional information and materials will be made available upon reasonable request.

Code availability. Any code used to analyse or plot data in this manuscript is available from the corresponding author upon request.

\section{Acknowledgments}

This work was financially supported by the National Key R\&D program of China (2019YFA0905602), the Chinese Academy of Sciences Strategic Priority Research Program (class B) (XDB38020300), and the National Natural Science Foundation of China (81773614).

\section{Author contributions}

Conceptualization, S.S. Q., H.W. L.; Methodology, S.S. Q., L. S., T. W., H.Q. D. and H.W. L.; Formal Analysis, S.S. Q., C. L. and K. W.; Investigation, S.S. Q., K. W., and L. B.; Resources, S.S. Q., and C. L.; Writing Original Draft, S.S. Q., and H. W. L.; Writing - Review \& Editing, S.S. Q., H.T. L. and H. W. L.; Supervision, S.J. L., and H.W. L.; Funding Acquisition, H.W. L.

\section{Competing interests}

The authors declare no competing interests. Patent related to this work, which numbers is CN2021107860719. 
Additional information

Extended data is available for this paper at https://doi.org/XX.

Supplementary information The online version contains supplementary material available at https://doi.org/XX.

Correspondence and requests for materials should be addressed to H.L.

\section{References}

1. Jagannathan, R., Patel, S. A., Ali, M. K. \& Narayan, K. M. V. Global Updates on Cardiovascular Disease Mortality Trends and Attribution of Traditional Risk Factors. Current Diabetes Reports 19, 44, doi:10.1007/s11892-019-1161-2 (2019).

2. Libby, P., Ridker, P. M. \& Hansson, G. K. Progress and challenges in translating the biology of atherosclerosis. Nature 473, 317-325, doi:10.1038/nature10146 (2011).

3. Libby, P. The changing landscape of atherosclerosis. Nature 592, 524-533, doi:10.1038/s41586-02103392-8 (2021).

4. Roy, P., Orecchioni, M. \& Ley, K. How the immune system shapes atherosclerosis: roles of innate and adaptive immunity. Nature Reviews Immunology, doi:10.1038/s41577-021-00584-1 (2021).

5. Bergheanu, S. C., Bodde, M. C. \& Jukema, J. W. Pathophysiology and treatment of atherosclerosis: Current view and future perspective on lipoprotein modification treatment. Neth Heart J 25, 231-242, doi:10.1007/s12471-017-0959-2 (2017).

6. Aherrahrou, R. et al. Genetic Regulation of Atherosclerosis-Relevant Phenotypes in Human Vascular Smooth Muscle Cells. Circulation Research 127, 1552-1565, doi:10.1161/circresaha.120.317415 (2020).

7. Goonewardena, S. N., Prevette, L. E. \& Desai, A. A. Metabolomics and Atherosclerosis. Current Atherosclerosis Reports 12, 267-272, doi:10.1007/s11883-010-0112-9 (2010).

8. Li, D. Y. \& Tang, W. H. W. Gut Microbiota and Atherosclerosis. Curr Atheroscler Rep 19, 39, doi:10.1007/s11883-017-0675-9 (2017).

9. Pan, H. Z. et al. Single-Cell Genomics Reveals a Novel Cell State During Smooth Muscle Cell Phenotypic Switching and Potential Therapeutic Targets for Atherosclerosis in Mouse and Human. Circulation 142, 2060-2075, doi:10.1161/circulationaha.120.048378 (2020).

10. Jonsson, A. L. \& Backhed, F. Role of gut microbiota in atherosclerosis. Nat Rev Cardiol 14, 79-87, doi:10.1038/nrcardio.2016.183 (2017).

11. Wang, Z. \& Zhao, Y. Gut microbiota derived metabolites in cardiovascular health and disease. Protein Cel/ 9, 416-431, doi:10.1007/s13238-018-0549-0 (2018).

12. Bennett, B. J. et al. Trimethylamine-N-oxide, a metabolite associated with atherosclerosis, exhibits complex genetic and dietary regulation. Cell Metab 17, 49-60, doi:10.1016/j.cmet.2012.12.011 
(2013).

13. Martin-Gallausiaux, C., Marinelli, L., Blottiere, H. M., Larraufie, P. \& Lapaque, N. SCFA: mechanisms and functional importance in the gut. Proceedings of the Nutrition Society 80, 37-49, doi:10.1017/s0029665120006916 (2021).

14. Wang, Y. F., Ding, W. X. \& Li, T. G. Cholesterol and bile acid-mediated regulation of autophagy in fatty liver diseases and atherosclerosis. Biochimica Et Biophysica Acta-Molecular and Cell Biology of Lipids 1863, 726-733, doi:10.1016/j.bbalip.2018.04.005 (2018).

15. Wang, K. et al. Structural Modification of Natural Product Ganomycin I Leading to Discovery of a alpha-Glucosidase and HMG-CoA Reductase Dual Inhibitor Improving Obesity and Metabolic Dysfunction in Vivo. J Med Chem 61, 3609-3625, doi:10.1021/acs.jmedchem.8b00107 (2018).

16. Qiao, S. S. et al. Activation of a Specific Gut Bacteroides-Folate-Liver Axis Benefits for the Alleviation of Nonalcoholic Hepatic Steatosis. Cell Reports 32, 108005, doi:10.1016/j.celrep.2020.108005 (2020).

17. Qiao, S. S. et al. The enriched gut commensal Faeciroseburia intestinalis contributes to the antimetabolic disorders effects of the Ganoderma meroterpene derivative. Food Science and Human Wellness 11, 85-96, doi:10.1016/j.fshw.2021.07.010 (2022).

18. Wang, K. et al. Parabacteroides distasonis Alleviates Obesity and Metabolic Dysfunctions via Production of Succinate and Secondary Bile Acids. Cell Rep 26, 222-235e225, doi:10.1016/j.celrep.2018.12.028 (2019).

19. Beckman, J. A., Creager, M. A. \& Libby, P. Diabetes and atherosclerosis: epidemiology, pathophysiology, and management. Jama 287, 2570-2581, doi:10.1001/jama.287.19.2570 (2002).

20. Stols-Gonçalves, D., Hovingh, G. K., Nieuwdorp, M. \& Holleboom, A. G. NAFLD and Atherosclerosis: Two Sides of the Same Dysmetabolic Coin? Trends in endocrinology and metabolism: TEM 30, 891902, doi:10.1016/j.tem.2019.08.008 (2019).

21. Wouters, K., Shiri-Sverdlov, R., van Gorp, P. J., van Bilsen, M. \& Hofker, M. H. Understanding hyperlipidemia and atherosclerosis: lessons from genetically modified apoe and Idlr mice. Clinical chemistry and laboratory medicine 43, 470-479, doi:10.1515/cclm.2005.085 (2005).

22. Li, T. et al. Defective Branched-Chain Amino Acid Catabolism Disrupts Glucose Metabolism and Sensitizes the Heart to Ischemia-Reperfusion Injury. Cell Metabolism 25, 374-385, doi:10.1016/j.cmet.2016.11.005 (2017).

23. Sun, H. P. et al. Catabolic Defect of Branched-Chain Amino Acids Promotes Heart Failure. Circulation 133, 2038-2049, doi:10.1161/circulationaha.115.020226 (2016).

24. Xu, Y., Jiang, H., Li, L., Chen, F. \& Liu, J. J. C. Branched-Chain Amino Acid Catabolism Promotes Thrombosis Risk by Enhancing Tropomodulin-3 Propionylation in Platelets. 142 (2020).

25. Kampoli, A. M., Tousoulis, D., Antoniades, C., Siasos, G. \& Stefanadis, C. Biomarkers of premature atherosclerosis. Trends in Molecular Medicine 15, 323-332, doi:10.1016/j.molmed.2009.06.001 (2009). 
26. Gorabi, A. M. et al. Implications for the role of lipopolysaccharide in the development of atherosclerosis. Trends in cardiovascular medicine, doi:10.1016/j.tcm.2021.08.015 (2021).

27. Soeki, T. \& Sata, M. Inflammatory Biomarkers and Atherosclerosis. International Heart Journal 57, 134-139, doi:10.1536/ihj.15-346 (2016).

28. Santilli, A. D., Dawson, E. M., Whitehead, K. J. \& Whitehead, D. C. Nonmicrobicidal Small Molecule Inhibition of Polysaccharide Metabolism in Human Gut Microbes: A Potential Therapeutic Avenue. ACS chemical biology 13, 1165-1172, doi:10.1021/acschembio.8b00309 (2018).

29. Douglas, G. M. et al. PICRUSt2 for prediction of metagenome functions. Nature Biotechnology 38 , 685-688, doi:10.1038/s41587-020-0548-6 (2020).

30. Wu, S. et al. GMrepo: a database of curated and consistently annotated human gut metagenomes. D1 (2019).

31. Lee, C. C. et al. Branched-Chain Amino Acids and Insulin Metabolism: The Insulin Resistance Atherosclerosis Study (IRAS). 582-588 (2016).

32. Tobias, D. K. et al. Circulating Branched-Chain Amino Acids and Incident Cardiovascular Disease in a Prospective Cohort of US Women. Circulation-Genomic and Precision Medicine 11, e002157, doi:10.1161/circgen.118.002157 (2018).

33. Bo, H. et al. A diabetes-predictive amino acid score and future cardiovascular disease.

34. Bhattacharya, S. et al. Validation of the association between a branched chain amino acid metabolite profile and extremes of coronary artery disease in patients referred for cardiac catheterization. Atherosclerosis 232, 191-196, doi:10.1016/j.atherosclerosis.2013.10.036 (2014).

35. Ridaura, V. K. et al. Gut Microbiota from Twins Discordant for Obesity Modulate Metabolism in Mice. Science 341, 1079-U1049, doi:10.1126/science.1241214 (2013).

36. Guo, C. J. et al. Depletion of microbiome-derived molecules in the host using Clostridium genetics. Science 366, e1331, doi:10.1126/science.aav1282 (2019).

37. Andreu, V. P., Roel-Touris, J., Dodd, D., Fischbach, M. A. \& Medema, M. H. The gutSMASH web server: automated identification of primary metabolic gene clusters from the gut microbiota. Nucleic Acids Research 49, W263-W270, doi:10.1093/nar/gkab353 (2021).

38. Jie, Z. et al. The gut microbiome in atherosclerotic cardiovascular disease. Nat Commun 8, 845, doi:10.1038/s41467-017-00900-1 (2017).

39. White, P. J. et al. Branched-chain amino acid restriction in Zucker-fatty rats improves muscle insulin sensitivity by enhancing efficiency of fatty acid oxidation and acyl-glycine export. Molecular Metabolism 5, 538-551, doi:10.1016/j.molmet.2016.04.006 (2016).

40. Zhou, M. Y. et al. Targeting BCAA Catabolism to Treat Obesity-Associated Insulin Resistance. Diabetes 68, 1730-1746, doi:10.2337/db18-0927 (2019).

41. Martinet, W., De Loof, H. \& De Meyer, G. R. Y. mTOR inhibition: A promising strategy for stabilization of atherosclerotic plaques. Atherosclerosis 233, 601-607, doi:10.1016/j.atherosclerosis.2014.01.040 (2014). 
42. Kurdi, A., De Meyer, G. R. Y. \& Martinet, W. Potential therapeutic effects of mTOR inhibition in atherosclerosis. British Journal of Clinical Pharmacology 82, 1267-1279, doi:10.1111/bcp.12820 (2016).

43. Charles et al. Endotoxin disrupts the leucine-signaling pathway involving phosphorylation of mTOR, 4E-BP1, and S6K1 in skeletal muscle. (2005).

44. Zhang, X. et al. High-protein diets increase cardiovascular risk by activating macrophage mTOR to suppress mitophagy. Nat. Metab. 2, 110-125, (2020).

45. Fingar, D. C. \& Blenis, J. J. O. Target of rapamycin (TOR): an integrator of nutrient and growth factor signals and coordinator of cell growth and cell cycle progression. 23, 3151 (2004).

46. Wang, Z. N. et al. Non-lethal Inhibition of Gut Microbial Trimethylamine Production for the Treatment of Atherosclerosis. Cell 163, 1585-1595, doi:10.1016/j.cell.2015.11.055 (2015).

47. Chen, P. B. et al. Directed remodeling of the mouse gut microbiome inhibits the development of atherosclerosis. Nature Biotechnology 38, 1288-1297, doi:10.1038/s41587-020-0549-5 (2020).

48. Kai Wang et al. A novel class of a-glucosidase and HMG-CoA reductase inhibitors from Ganoderma leucocontextum and the anti-diabetic properties of ganomycin I in KK-A y mice. 127, 1035-1046 (2017).

49. Wu, T. R. et al. Gut commensal Parabacteroides goldsteinii plays a predominant role in the antiobesity effects of polysaccharides isolated from Hirsutella sinensis. Gut 68, 248-262, doi:10.1136/gutjnl-2017-315458 (2019).

50. Sato, Y. et al. Novel bile acid biosynthetic pathways are enriched in the microbiome of centenarians. Nature, https://doi.org/10.1038/s41586-41021-03832-41585 doi:10.1038/s41586-021-03832-5 (2021).

51. Olson, C. A. et al. The Gut Microbiota Mediates the Anti-Seizure Effects of the Ketogenic Diet. Cell $173,1728-$

1741.e1713

, doi:10.1016/j.cell.2018.04.027 (2018).

52. Li, J., Lin, S. Q., Vanhoutte, P. M., Woo, C. W. \& Xu, A. M. Akkermansia Muciniphila Protects Against Atherosclerosis by Preventing Metabolic Endotoxemia-Induced Inflammation in Apoe(-/-) Mice. Circulation 133, 2434-2446, doi:10.1161/circulationaha.115.019645 (2016).

53. Neinast, M. D. et al. Quantitative Analysis of the Whole-Body Metabolic Fate of Branched-Chain Amino Acids. Cell Metabolism 29, 417-429.e414, doi:10.1016/j.cmet.2018.10.013 (2019).

54. Pedersen, H. K. et al. Human gut microbes impact host serum metabolome and insulin sensitivity. Nature 535, 376-381, doi:10.1038/nature18646 (2016).

55. Oh, S. F. et al. Host immunomodulatory lipids created by symbionts from dietary amino acids. Nature, doi:10.1038/s41586-021-04083-0 (2021).

56. Yu, D. Y. et al. The adverse metabolic effects of branched-chain amino acids are mediated by isoleucine and valine. Cell Metabolism 33, 905-922.e906, doi:10.1016/j.cmet.2021.03.025 (2021). 
57. Heimann, E., Nyman, M., Palbrink, A. K., Lindkvist-Petersson, K. \& Degerman, E. Branched short-chain fatty acids modulate glucose and lipid metabolism in primary adipocytes. Adipocyte 5, 359-368, doi:10.1080/21623945.2016.1252011 (2016).

58. Bellono, N. W. et al. Enterochromaffin Cells Are Gut Chemosensors that Couple to Sensory Neural Pathways. Cell 170, 185-

198.e116 , doi:10.1016/j.cell.2017.05.034 (2017).

59. Liu, C., Du, M., Abuduaini, R., Yu, H. Y. \& Liu, S. J. J. M. Enlightening the Taxonomy Darkness of Human Gut Microbiomes With a Cultured Biobank. 9 (2021).

60. Masella, A. P., Bartram, A. K., Truszkowski, J. M., Brown, D. G. \& Neufeld, J. D. PANDAseq: PAired-eND Assembler for Illumina sequences. Bmc Bioinformatics 13, 31, doi:10.1186/1471-2105-13-31 (2012).

61. Love, M. I., Huber, W. \& Anders, S. Moderated estimation of fold change and dispersion for RNA-seq data with DESeq2. Genome Biology 15, doi:10.1186/s13059-014-0550-8 (2014).

62. Beghini, F., Mciver, L. J., Blanco-Míguez, A., Dubois, L. \& Segata, N. J. e. Integrating taxonomic, functional, and strain-level profiling of diverse microbial communities with bioBakery 3. 10, e65088 (2021).

63. Huson, D. H. \& Buchfink, B. J. N. M. Fast and sensitive protein alignment using DIAMOND. (2015).

64. Derrick, Wood, Steven \& Biology, S. J. G. Kraken: ultrafast metagenomic sequence classification using exact alignments. (2014).

\section{Figures}


a
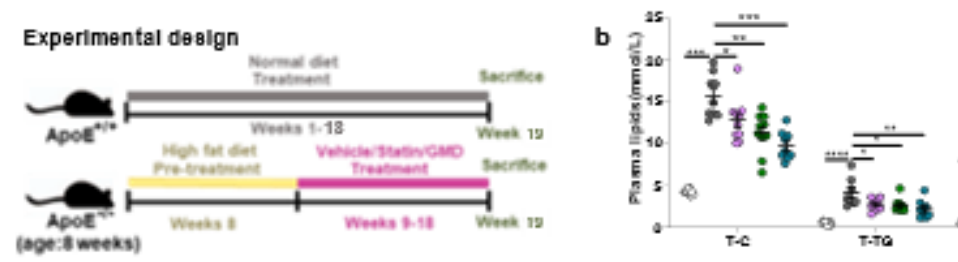

$\therefore$ Apot $E^{N+N D}$

- Apot HFo-Butn

- Apde HFo Como

c
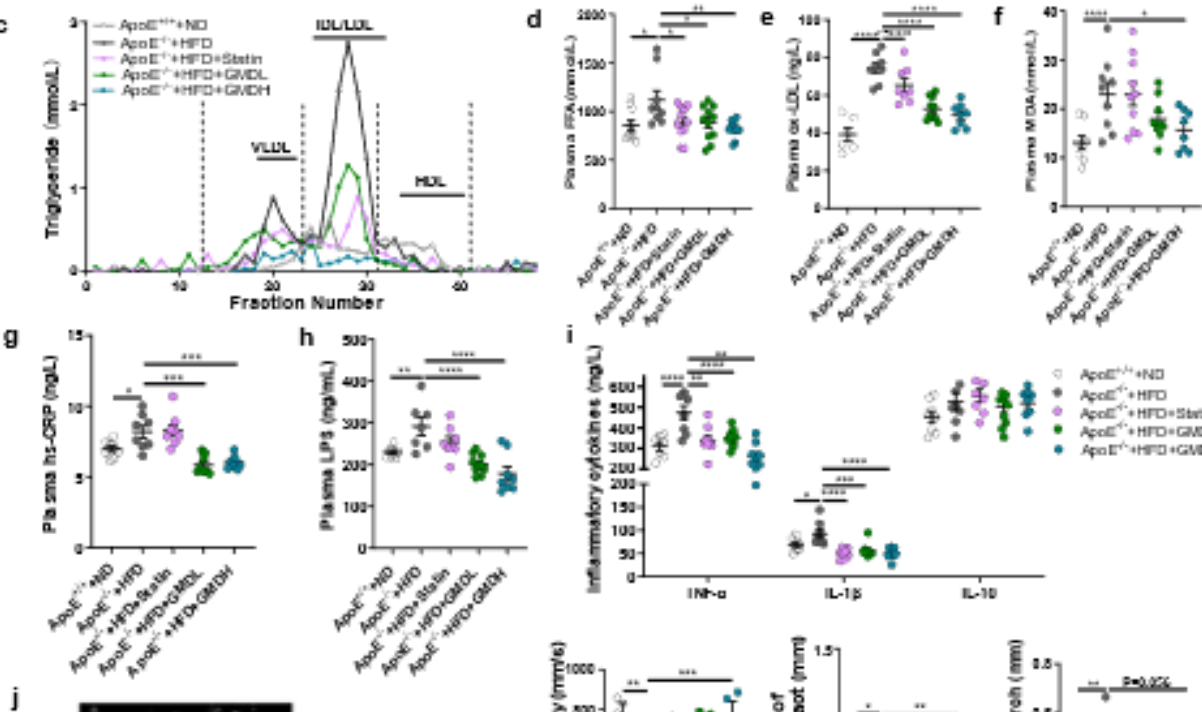

j
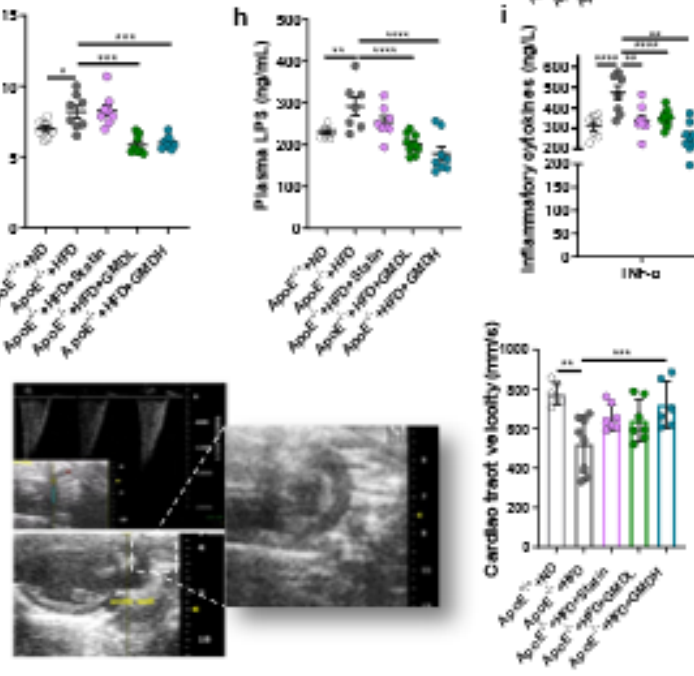

$\frac{\frac{1}{m}}{\frac{7}{3}}$

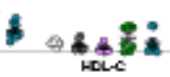

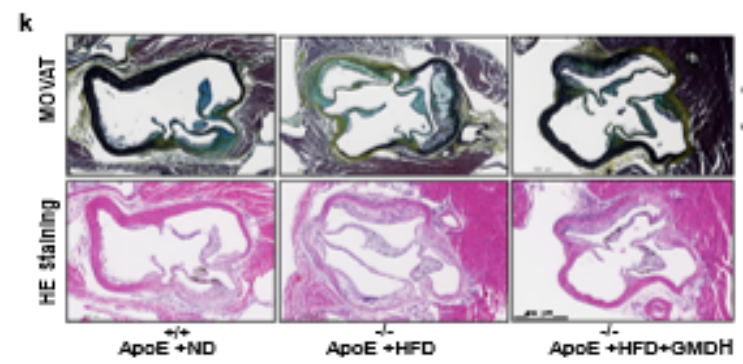

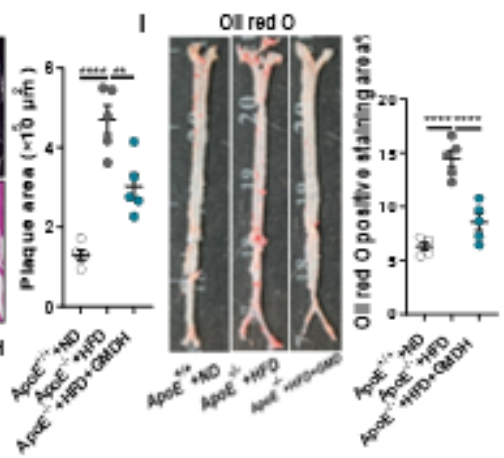

\section{Figure 1}

HFD-fed ApoE-/- mice treated with GMD exhibit reduced atherosclerosis. a, Experimental design showing groups and durations. b, Plasma T-C, T-TG, LDL-C and HDL-C levels. c, FPLC analysis of plasma triglyceride levels. d, Plasma FFA. e, Plasma ox-LDL. f, Plasma MDA. g, Plasma hs-CRP. h, Plasma LPS. i, Inflammation cytokines. ( $n=9-10$ mice per group). j, Quantitative analysis of blood flow velocity by ultrasound biomicroscopy (UBM) (top left); image showing wall of ascending aorta (bottom left). Quantitative analysis of the cardiac tract velocity, quantitative thickness analysis of cardiac outflow tract and quantitative thickness analysis of the aortic arch by vascular ultrasound (right) ( $n=5-8$ mice per 
group). $k$, Representative MOVAT and H\&E staining of cross-sections of aortic roots and quantitative data ( $n=5$ total samples analyzed per group) (scale bar $=400 \mu \mathrm{m}$ ). I, Lipid content of whole aorta visualized by staining with oil red 0 , and quantitative data ( $\mathrm{n}=5$ total samples analyzed, per group). Movat pentachrome stain of a cross-section of one of the aortic roots shown in (k); cardiomyocytes (red), elastic tissue (black), arterial plaque (yellow-green), foam cells (purple), collagen (yellow). Data are mean \pm s.e.m. Statistical analysis was performed using one-way ANOVA followed by the Tukey post hoc test. * $\mathrm{P}<0.05$; $* * \mathrm{P}<0.01$; *** $\mathrm{P}<0.001$; $* * * * \mathrm{P}<0.0001$; exact $\mathrm{P}$ values are provided in the Source Data.

a

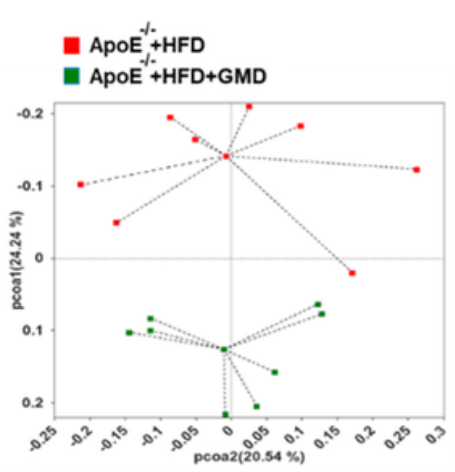

b

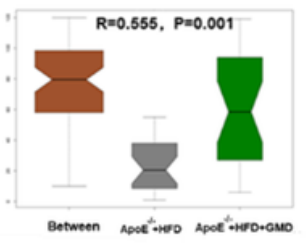

e

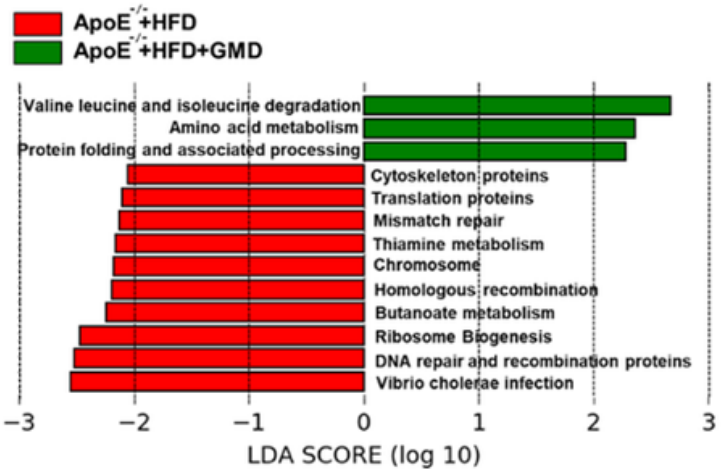

C Cladogram

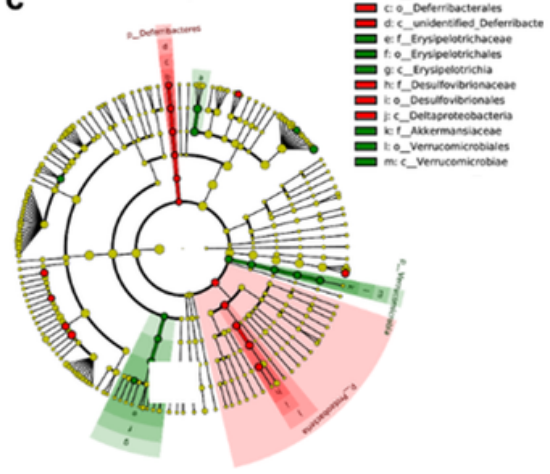

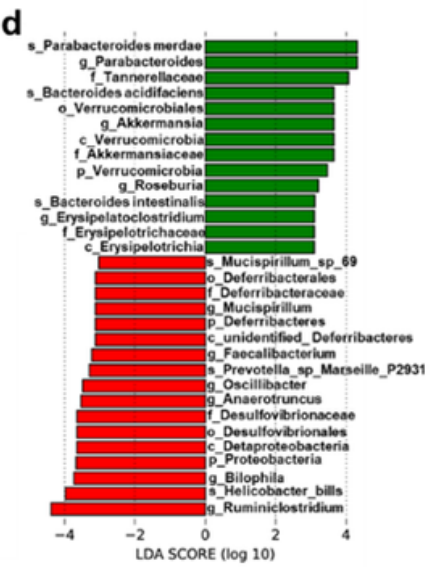

$\mathbf{f}$
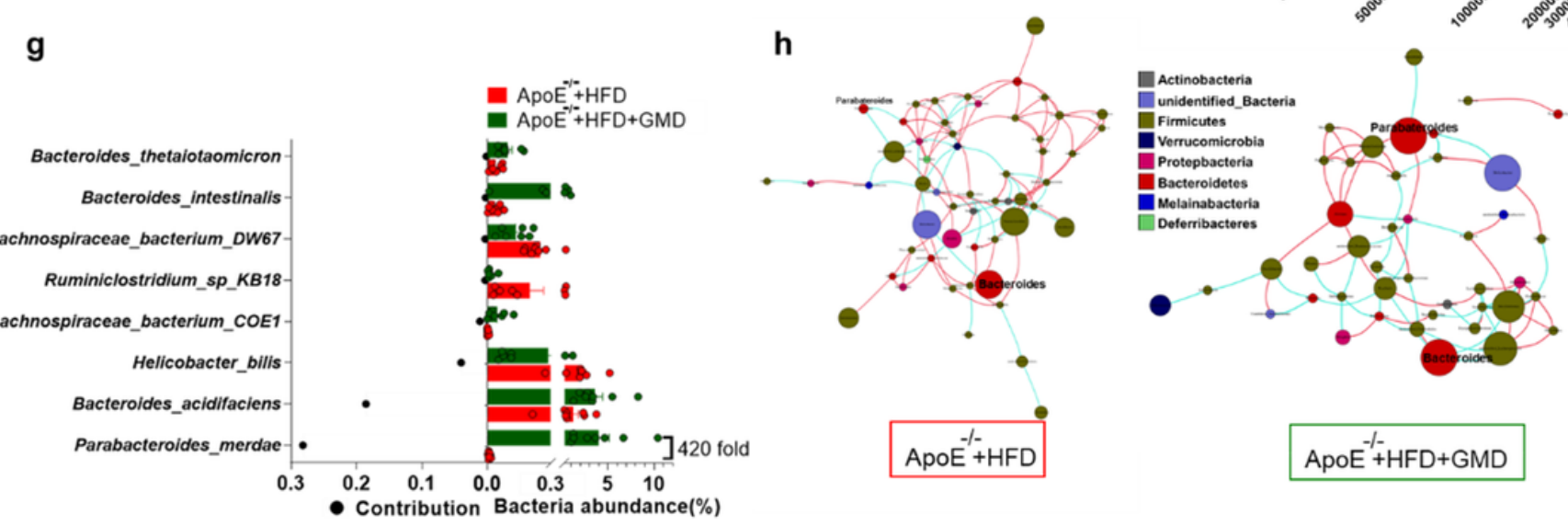

Figure 2

GMD-modulated changes in the HFD-fed ApoE-/- mice microbiota. 16S rRNA gene sequence data comparing the caecal microbiota of vehicle or GMD $(10 \mathrm{mg} / \mathrm{kg})$ treatment ApoE-/- mice. a, Principal coordinate analysis (PCoA) of all samples by weighted UniFrac distance. b, Analysis of similarities 
(ANOSIM) test to compare R values between different groups. The size of nodes was proportional to the relative abundance of genera. $c$, Taxonomic cladogram generated from linear discriminant analysis effect size (LEfSe) analysis of 16S rRNA gene sequences. d, LDA score representing the taxonomic data with significant difference between ApoE-/-+HFD and ApoE-/-+HFD +GMD groups. Only LDA scores $>3$ are shown. Green indicates enriched taxa in the ApoE-/-+HFD +GMD group. Red indicates enriched taxa in the ApoE-/-+HFD group. Each circle's size is proportional to the taxon's abundance. e, Relative abundance of metabolic pathways (LDA>2) in gut predicted by PICRUSt2 and analyzed by LEfSe. $f$, Differences in microbial functions associated with amino acid metabolism between the ApoE-/-+HFD and ApoE-/-+HFD +GMD groups based on KEGG functional categories (Wilcoxon rank-sum test and Storey's methods for multiple tests adjustment). g, Results of similarity percentage (SIMPER) analysis to quantify the contribution of top eight significantly different species between the two groups and the abundances (percent reads) of these species based on 16S rRNA gene sequence data. h, Gut microbial co-occurrence network analysis based on core genus (average relative abundance $>0.1 \%$ ) in the ApoE-/-+HFD and ApoE-/-+HFD +GMD groups. Red line: Spearman's rank correlation coefficient $>0.30$; blue line: Spearman's rank correlation coefficient $<-0.30 . n=8$ mice per group. For box plots, interleaved box and whiskers represent min to max, Mann-Whitney $U$ test $(H) * P<0.05$; $* * \quad P<0.01$; $* \star * ~ P<0.001$; exact $P$ values are provided in the Source Data. 


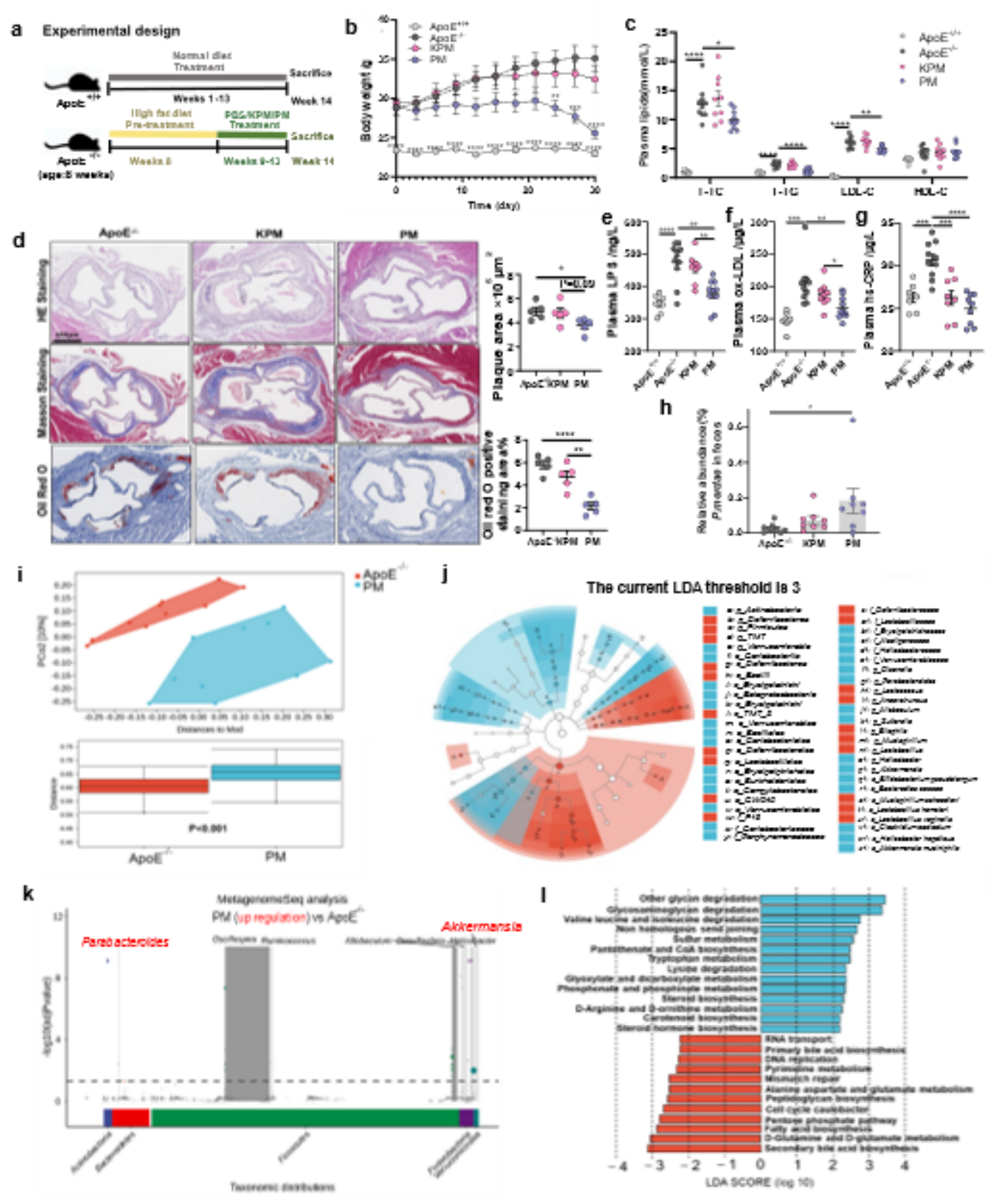

\section{Figure 3}

Gavage with live P. merdae attenuates the formation of atherosclerotic plaque. a, Experimental design showing groups and durations. b, Body weight. c, Plasma T-C, T-TG, LDL-C and HDL-C levels. d, Representative H\&E staining, Masson, and oil red O staining of cross-sections of aortic roots, scale bar = $400 \mu \mathrm{m}$, and quantitative data ( $\mathrm{n}=5$ total samples analyzed, per group); Masson staining of a crosssection of one of the aortic roots shown in (d), cardiomyocytes (red), nucleus (blue black), collagen (blue); e, Plasma LPS. f, Plasma ox-LDL. g, Plasma hs-CRP. h, Colonization of P. merdae strain in the gut of ApoE-/- mice. The relative abundance in feces was determined post three days of four-week treatment by qPCR. Data are mean \pm s.e.m. Statistical analysis was performed using one-way ANOVA followed by the 


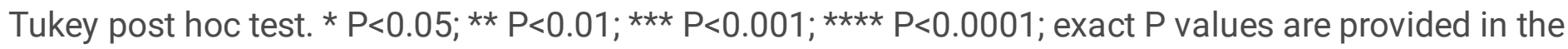
Source Data. i, Principal coordinate analysis (PCoA) of all samples by weighted UniFrac distance. j,

Taxonomic cladogram generated from linear discriminant analysis effect size (LEfSe) analysis of $16 \mathrm{~S}$ rRNA gene sequences. LDA score representing the taxonomic data with significant differences between ApoE-/- and PM groups. Only LDA scores $>3$ are shown. $k$, Genus with significant difference between ApoE-/- and PM groups in Metagenome Seq analysis. I, Relative abundance of metabolism pathways $(\mathrm{LDA}>2)$ in gut predicted by PICRUSt2 and analyzed by LEfSe. Abbreviation: ApoE+/+, C57BL/6J mice; ApoE-/-, HFD-fed ApoE-/- mice; KPM, heat-killed P. merdae; PM, P. merdae. $n=$ 8-9 mice per group.
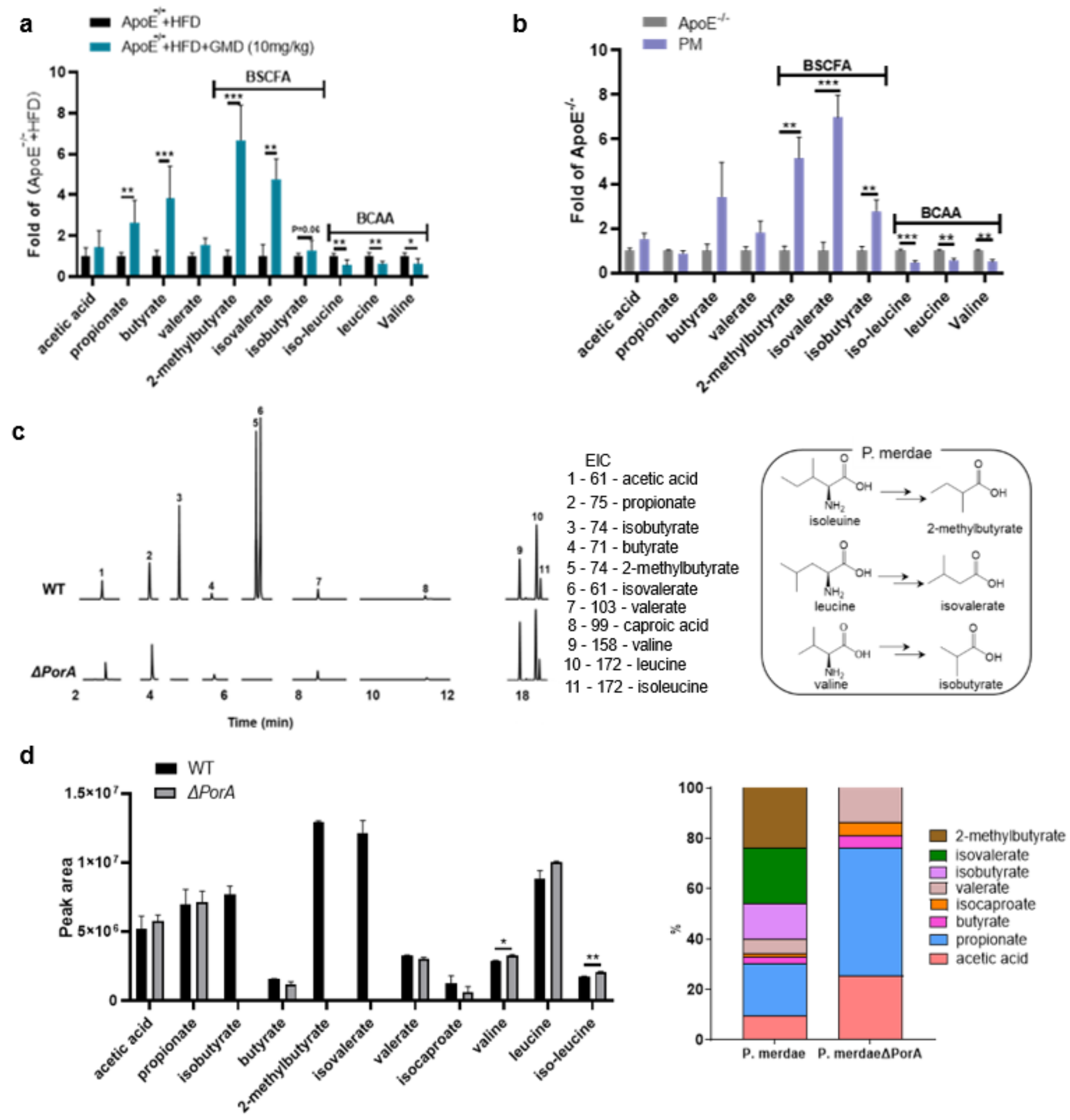


\section{Figure 4}

Identification of branched-chain amino acid degradation pathways in P. merdae. a, b, In vivo levels of BCAAs and SCFAs in feces in ApoE-/-+HFD vs ApoE-/-+HFD +GMD mice (a), and ApoE-/-vs PM groups (b), (one technical replicate of six biological replicates for each group). c, P. merdae mutant exhibits specific loss of BSCFA production in vitro (left) and BCAA metabolic pathway from P. merdae examined in this study (right). d, P. merdae and P. merdae mutant were cultured and assayed for SCFA metabolites by GC-MS in vitro (one technical replicate of three biological replicates for each group). Data are mean \pm s.e.m. Statistical analysis was performed using two-sided Student's t-test (Gaussian model) test. * $P<0.05$; $* \star P<0.01 ; * \star \star ~ P<0.001$; exact $P$ values are provided in the Source Data. 
a

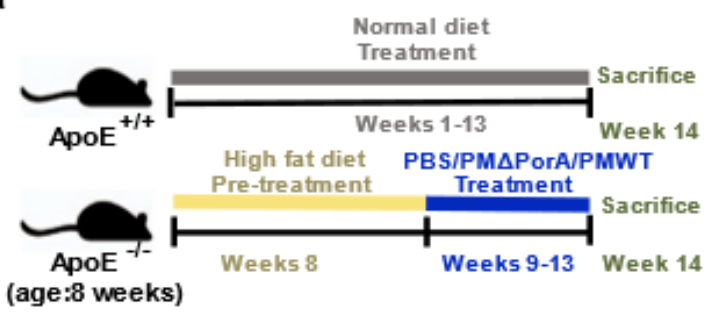

b

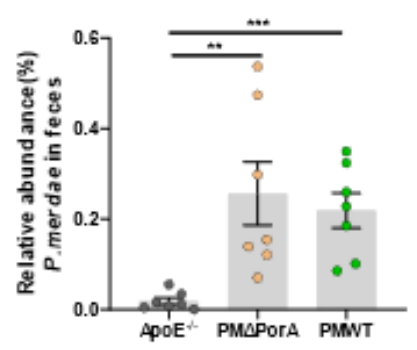

C

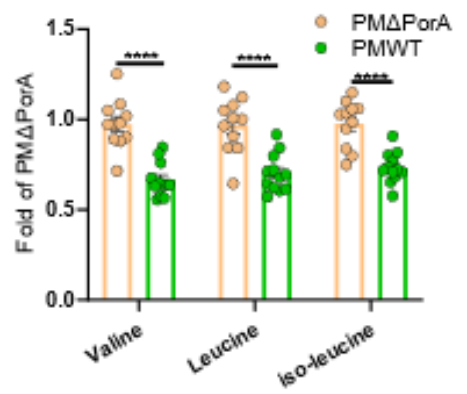

e

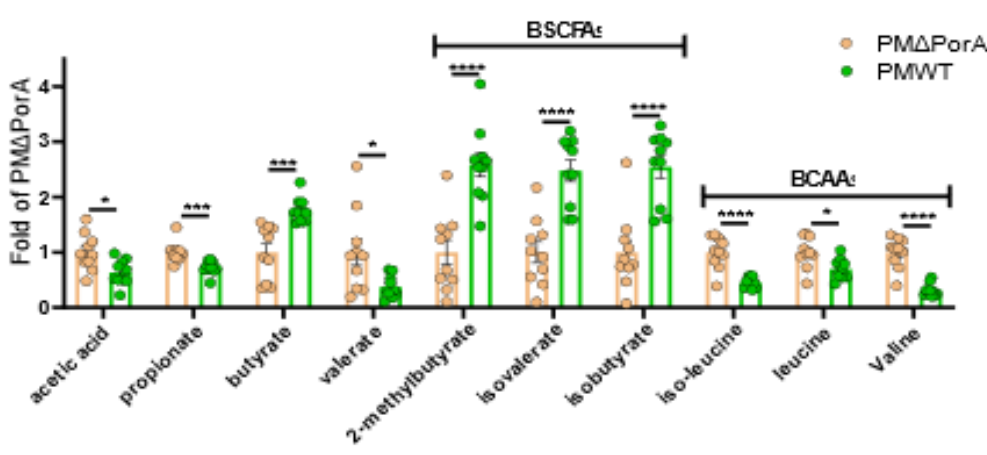

f

g

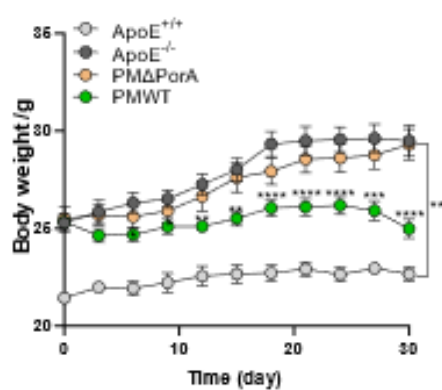

h

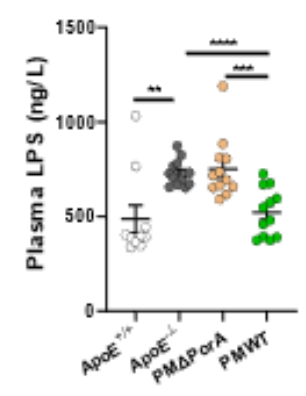

i

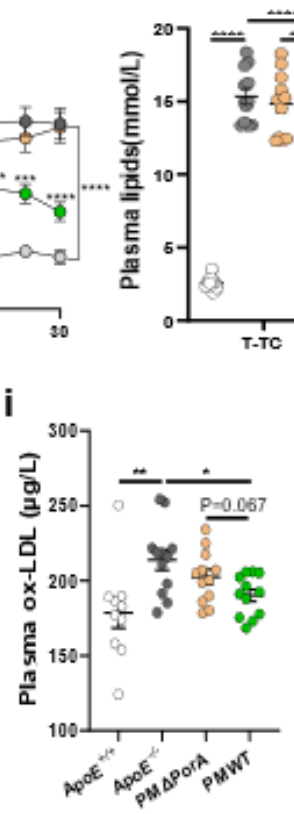

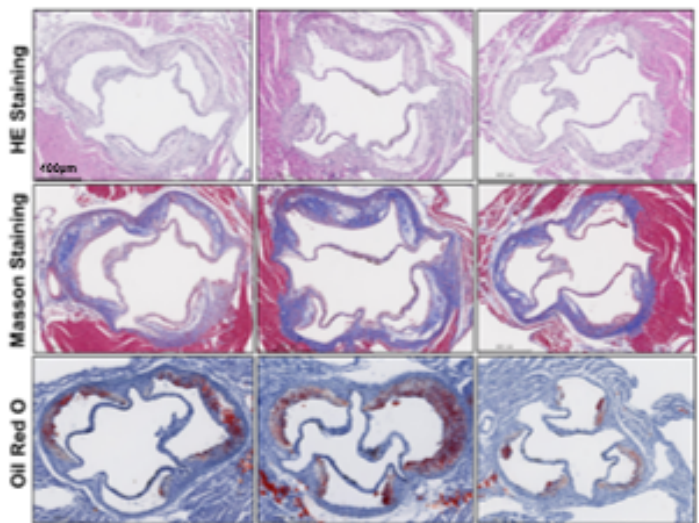

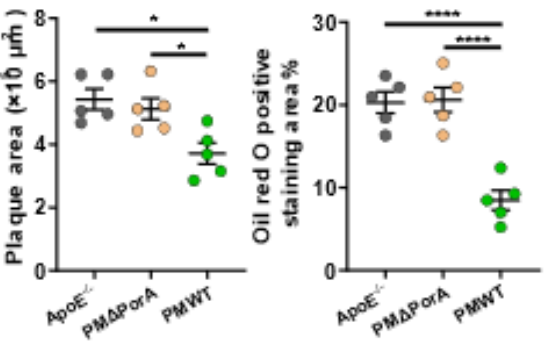

\section{Figure 5}

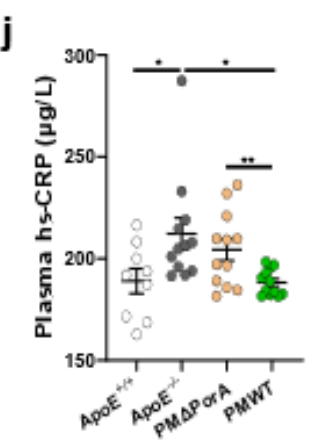

k

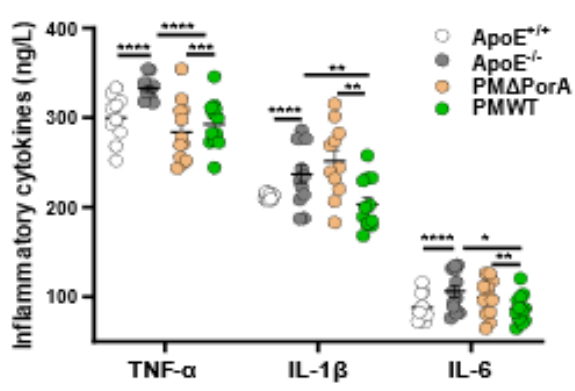

Increased BCAA degradation by P. merdae attenuates the formation of atherosclerotic plaque in HFD-fed ApoE-/- mice. a, Experimental design. b, Colonization of PMWT and PM $\triangle$ PorA strains in the gut of ApoE-/mice. The relative abundance of PMWT (green) and PM $\triangle$ PorA (orange) in feces were determined post three days of four-week treatment by qPCR ( $n=7$ mice per group). $c$, The levels of BCAAs in plasma. $d$, The levels of BCAAs and SCFAs in feces. e, Representative H\&E, Masson, and oil red 0 staining of crosssections of aortic roots, scale bar $=400 \mu \mathrm{m}$, and quantitative data $(\mathrm{n}=5$ total samples analyzed per group); Masson staining of a cross-section of one of the aortic roots shown in (e), cardiomyocytes (red), 
nucleus (blue black), collagen (blue). f, Body weight. g, Plasma T-C, T-TG, LDL-C and HDL-C levels. $h$, Plasma LPS. i, Plasma ox-LDL. j, Plasma hs-CRP. Abbreviation: ApoE+/+, C57BL/6J mice; ApoE-/-, HFDfed ApoE-/- mice; PM $\triangle$ PorA, P. merdae with knockout of porA gene; PMWT, wild type P. merdae. $n=12$ mice per group. Data are mean \pm s.e.m. Statistical analysis was performed using one-way ANOVA

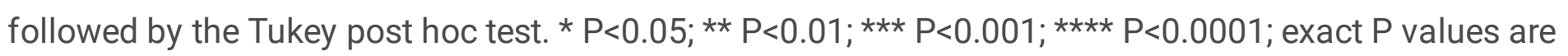
provided in the Source Data.

a

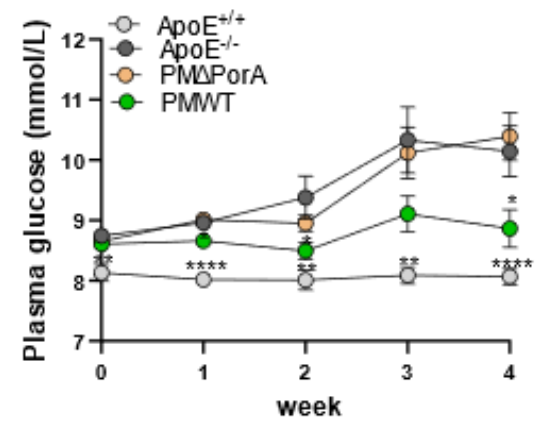

b

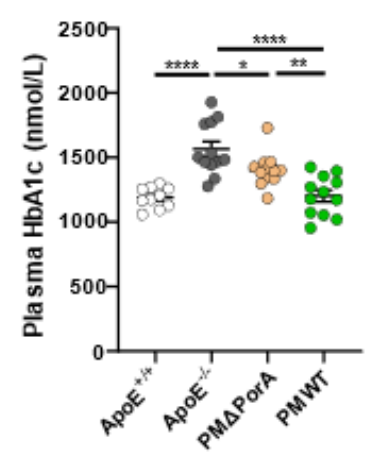

C

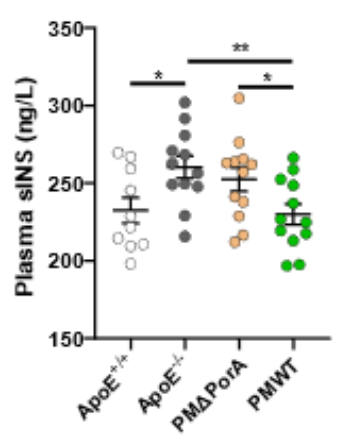

d

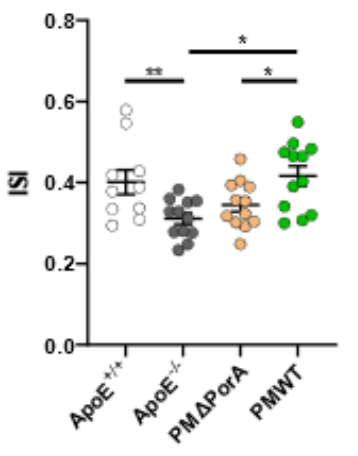

e

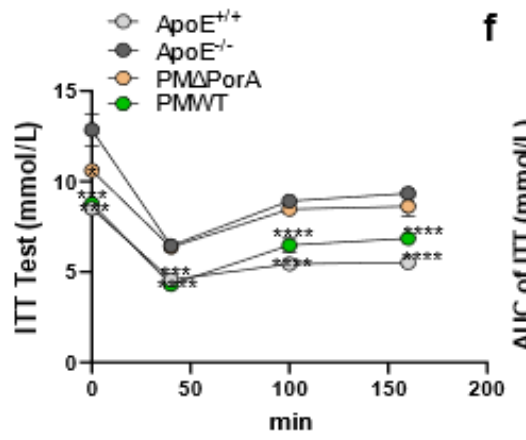

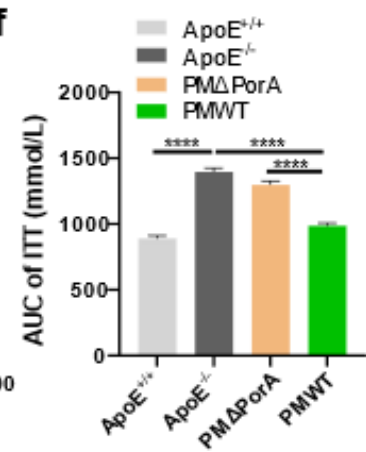

g
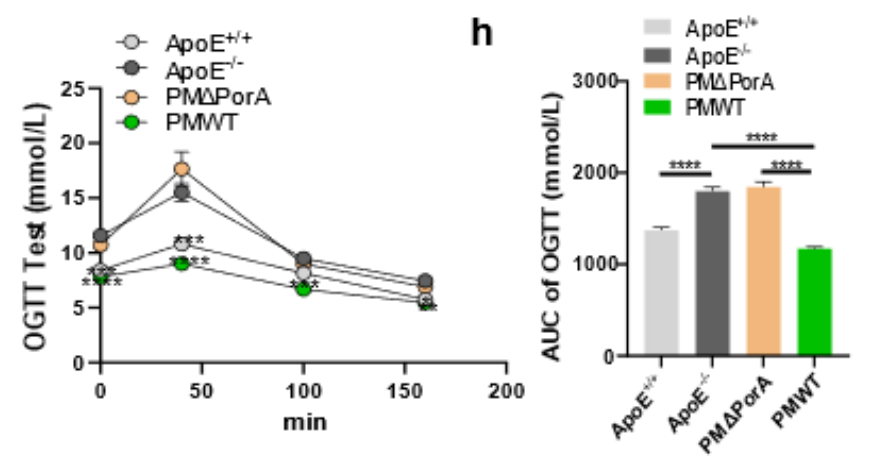

i
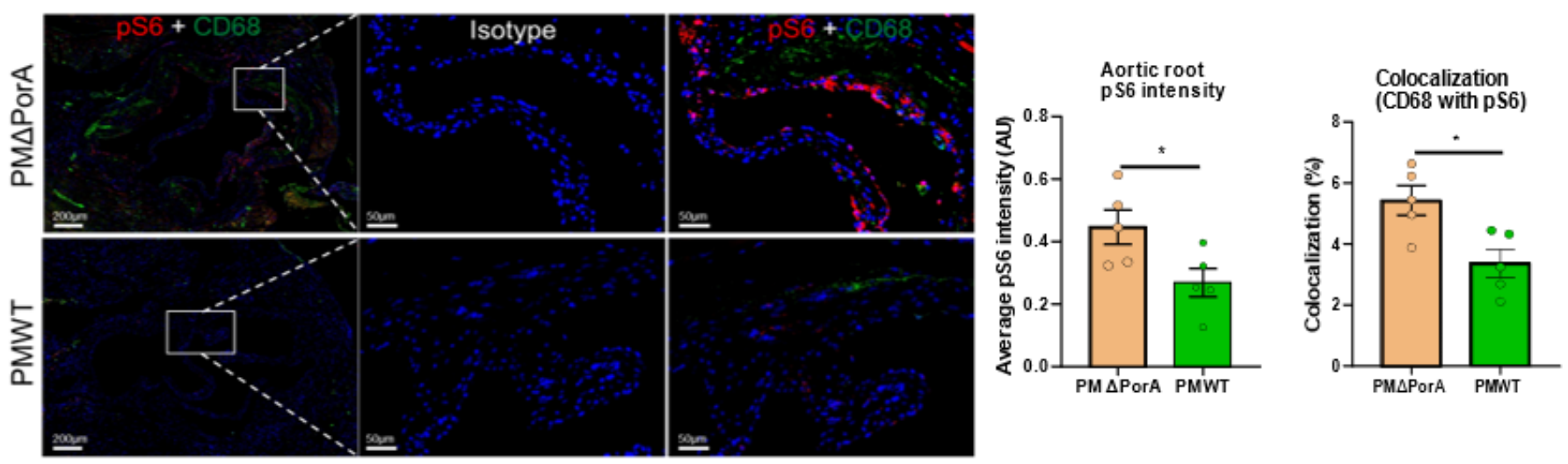

\section{Figure 6}

Gut BCAA degradation by P. merdae regulates plaque macrophage mTORC1 signaling. (A) Free diet plasma glucose. (B) Plasma HbA1c. (C) Plasma sINS. (D) Insulin sensitivity index (ISI). (E) ITT test. (F) AUC of ITT. (G) OGTT test. (H) AUC of OGTT. (I) Immunostaining of pS6 levels and colocalization of pS6 (red) with the macrophage marker CD68 (green) in atherosclerotic plaques. Representative images are shown on the left and quantification on the right. AU, arbitrary units. Scale bar = $100 \mu \mathrm{m}$. (J) Plasma TNF- 
a. (K) Plasma IL-1ß. (J) Plasma IL-6. Abbreviation: ApoE-/-, HFD-fed ApoE-/- mice; PM $\triangle$ PorA, P. merdae with knockout of porA gene; PMWT, wild type P. merdae. Data are mean \pm SEM. Statistical analysis was performed using one-way ANOVA followed by the Tukey post hoc test. * $P<0.05$; ${ }^{* \star} P<0.01$; ${ }^{* \star \star} P<0.001$; $\star \star \star \star P<0.0001$. exact $P$ values are provided in the Source Data.

\section{Supplementary Files}

This is a list of supplementary files associated with this preprint. Click to download.

- HongweiLiuSI.docx 